\title{
Analysis of triglyceride synthesis unveils a green algal soluble diacylglycerol acyltransferase and provides clues to potential enzymatic components of the chloroplast pathway
}

Carolina Bagnato ${ }^{1}$, María B. Prados ${ }^{1}$, Gisela R. Franchini², Natalia Scaglia ${ }^{2}$, Silvia E. Miranda ${ }^{3}$ and María V. Beligni ${ }^{4^{*}}$

\begin{abstract}
Background: Microalgal triglyceride (TAG) synthesis has attracted considerable attention. Particular emphasis has been put towards characterizing the algal homologs of the canonical rate-limiting enzymes, diacylglycerol acyltransferase (DGAT) and phospholipid:diacylglycerol acyltransferase (PDAT). Less work has been done to analyze homologs from a phylogenetic perspective. In this work, we used HMMER iterative profiling and phylogenetic and functional analyses to determine the number and sequence characteristics of algal DGAT and PDAT, as well as related sequences that constitute their corresponding superfamilies. We included most algae with available genomes, as well as representative eukaryotic and prokaryotic species.

Results: Amongst our main findings, we identified a novel clade of DGAT1-like proteins exclusive to red algae and glaucophyta and a previously uncharacterized subclade of DGAT2 proteins with an unusual number of transmembrane segments. Our analysis also revealed the existence of a novel DGAT exclusive to green algae with moderate similarity to plant soluble DGAT3. The DGAT3 clade shares a most recent ancestor with a group of uncharacterized proteins from cyanobacteria. Subcellular targeting prediction suggests that most green algal DGAT3 proteins are imported to the chloroplast, evidencing that the green algal chloroplast might have a soluble pathway for the de novo synthesis of TAGs. Heterologous expression of $C$. reinhardtii DGAT3 produces an increase in the accumulation of TAG, as evidenced by thin layer chromatography.
\end{abstract}

Conclusions: Our analysis contributes to advance in the knowledge of complex superfamilies involved in lipid metabolism and provides clues to possible enzymatic players of chloroplast TAG synthesis.

Keywords: Algae, Biodiesel production, Chloroplast, HMMER profiling, Neutral lipids, Soluble acyltransferase, Protein phylogeny, Triglyceride metabolism

\footnotetext{
* Correspondence: mvbeligni@mdp.edu.ar

${ }^{4}$ Instituto de Investigaciones Biológicas (IIB-CONICET-UNMdP), Facultad de Ciencias Exactas y Naturales, Universidad Nacional de Mar del Plata, CC 1245, 7600 Mar del Plata, Argentina

Full list of author information is available at the end of the article
} 


\section{Background}

There is currently great recognition of the potential of microalgae for the production of biodiesel. However, the difficulties in maintaining elevated culture biomass and oil productivity create bottlenecks that hamper the economic viability of the process [1-3]. Considerable work has been done to improve the conditions of microalgal culture in order to increase oil production [4-8]. A concluding remark of many experiments is that most species increase their triglyceride (TAG) content under stress, such us nitrogen deprivation [9-12]. The challenge is that TAG accumulation seems to occur in conjunction with a reduction in cell proliferation, with a consequent decrease in total oil productivity. In this scenario, genetic engineering of lipid metabolism could constitute a powerful tool to overcome these limitations. However, following this route is not trivial, since a profound understanding of lipid metabolic pathways is required in order to tailor genetic engineering tools. This is particularly true for algae, organisms for which our knowledge about TAG metabolism is incomplete and mostly inferred. First, algae are a paraphyletic group with representatives of several different kingdoms or supergroups [13]. Second, not all algae accumulate TAGs as their main storage compound [14-16], hence it is expected that this creates fundamental differences in the regulation of the synthesizing pathways. Third, even for the more-closely related green algae, recent studies have put in evidence interesting differences between microalgal and plant TAG metabolism $[17,18]$.

TAGs are the major molecules of energy storage in most eukaryotes. There are several accepted pathways for the synthesis of TAGs. One of the best characterized is the Kennedy pathway, which implies the sequential acylation of fatty acids (usually activated in the form of acyl-CoA) on a glycerol-3-phosphate backbone. In animals, yeasts and plants, in which it has been best characterized, this pathway is located in the endoplasmic reticulum, evidenced by subcellular localization studies of the main participating enzymes [19-21]. The final step, the acylation of acyl-CoA onto a molecule of sn1,2-diacylglycerol (DAG) is the rate-limiting step, and is catalyzed by several types of diacylglycerol acyltransferases (DGATs). Most DGATs are integral microsomal membrane proteins. The most numerous and best characterized forms in mammals [22, 23] and plants [21] are DGAT1 and DGAT2, which, despite sharing a few amino acids, show overall little sequence similarity with one another. In addition, other DGAT forms were reported in particular tissues of certain plants [24-26].

In yeasts and plants, TAG biosynthesis can also occur via phospholipid (PL) remodeling, via the action of CoA-independent transacylases [27]. The best characterized is membrane-bound phospholipid: diacylglycerol acyltransferase (PDAT). PDAT catalyzes the transfer of a fatty acyl moiety from the sn-2-position of a PL to the sn-3-position of sn-1, 2- DAG, thus forming TAG and a lyso-phospholipid [27, 28].

Several structural and evolutionary studies have been done for DGATs [29-32] and PDATs [27, 33], but only a scarce amount have tried to analyze algal homologs from different supergroups in a phylogenetic context [34, 35]. One of the key steps towards characterizing microalgal TAG synthesis pathway refers to the proper annotation of enzymes. Most enzymes are initially identified through in silico similarity searches using canonical enzymes with biochemical evidence as templates. Most of these enzymes are from plants, yeasts and animals, which not always represent the best dataset to search for homologs in unrelated organisms, such as heterokonts. In addition, the tools most commonly used, such as BLAST, use limited information to perform a search and are not always effective at identifying distant homologs. Other tools, such as HMMER, PHI-BLAST or a combination of methods in an iterative form, include more parameters into the search and are, hence, more sensitive than BLAST [36-38].

With the purpose of identifying all possible enzymes within the TAG synthesis pathway in algae and analyzing them in a phylogenetic context, we performed an in-depth sequence data mining. We followed a HMMER-iterative strategy that included 26 algae and 53 related and non-related eukaryotic species, as well as representative prokaryotic species. Phylogeny and standard biocomputational analyses were performed in order to make testable predictions of the characteristics of the encoded proteins in different algae taxa. Our results show some interesting new findings about several of the enzymes analyzed. One of the most significant refers to the identification of a novel DGAT exclusive to green algae that shows moderate similarity to DGAT3, a soluble DGAT previously characterized in plants $[25,39,40]$. Subcellular localization prediction suggests that most green algal DGAT3 proteins likely localize to the chloroplast. Phylogenetic analysis evidences that the DGAT3 clade shares a most recent ancestor with a group of uncharacterized proteins from cyanobacteria. Heterologous expression of $C$. reinhardtii DGAT3 produced an increase in the accumulation of TAGs in E. coli cells compared to control bacteria. Altogether, our analysis provides useful information about the characteristics and phylogenetic relationships of the main enzymes that participate in TAG synthesis. The implications of the existence of a green algal DGAT3, as well as its possible involvement in chloroplast TAG synthesis, is discussed. 


\section{Results}

With the purpose of annotating the TAG synthesis pathway in algae following a phylogenetic perspective and trying to identify novel homologs, we did a HMMER iterative data mining. We used the predicted proteomes from most algae with available sequenced genomes (Table 1), as well as from a set of representative eukaryotic organisms (Additional file 1). In order to do the analyses in a true phylogenetic context, we also searched for prokaryotic homologs in the HMMER website. Although this procedure was done for PDAT and for all the enzymes of the de novo synthesis of TAGs, we mainly focused on the enzymes of the rate-limiting steps, PDAT and DGATs.

\section{PDAT in-silico analyses}

PDATs (EC:2.3.1.158) belong to a superfamily that is also composed of lecithin:cholesterol acyltransferases (LCATs) (EC: 2.3.1.43), enzymes that catalyze the transacylation of acyl groups from phospholipids to a variety of sterols [33]. Arabidopsis thaliana has two reported PDATs (PDAT1 and PDAT2) [28] and four LCAT-like proteins. LCAT2 is a

Table 1 Algae species used for computational analysis of the TAG pathway

\begin{tabular}{|c|c|c|c|}
\hline \multicolumn{2}{|c|}{ Classification at Higher Ranks $^{a}$} & \multirow{2}{*}{$\begin{array}{l}\text { Lower Ranks } \\
\text { Cyanophora paradoxa }\end{array}$} & \multirow{2}{*}{$\frac{\text { Code }^{t}}{\text { Cypa }}$} \\
\hline ARCHAEPLASTIDA & Glaucophyta & & \\
\hline & Rhodophyta (Red Algae) & Cyanidioschyzon merolae & Cmer \\
\hline & & Galderia sulphuraria & Gsul \\
\hline & & Chondrus crispus & Ccri \\
\hline & & Porphyridium purpureum & Ppur \\
\hline & Chloroplastida & Chlorophyta (Green Algae) & \\
\hline & & Coccomyxa subellipsoidea & Csub \\
\hline & & Chlorella variabilis & Cvar \\
\hline & & Chlamydomonas reinhardtii & Crei \\
\hline & & Volvox carteri & Vcar \\
\hline & & Bathycoccus prasinos & Bpra \\
\hline & & Micromonas pusilla & Mpus \\
\hline & & Micromonas sp. & Misp \\
\hline & & Ostreococcus lucimarinus & Oluc \\
\hline & & Ostreococcus tauri & Otau \\
\hline & & Streptophyta (Plants) & \\
\hline \multirow[t]{15}{*}{ SAR } & Rhizaria & Bigelowiella natans & Bnat \\
\hline & Alveolata & Dinoflagellata (Dinoflagellates) & \\
\hline & & Symbiodinium minutum & Smin \\
\hline & Stramenopiles & Eustigmatales & \\
\hline & & Nannochloropsis gaditana & Ngad \\
\hline & & Nannochloropsis oceanica & Noce \\
\hline & & Pelagophyceae & \\
\hline & & Aureococcus anophagefferens & Aano \\
\hline & & Phaeophyceae (Brown Algae) & \\
\hline & & Ectocarpus siliculosus & Esil \\
\hline & & Diatomea (Diatoms) & \\
\hline & & Fragilariopsis cylindrus & Fcyl \\
\hline & & Pseudo-nitzschia multiseries & Pmul \\
\hline & & Phaeodactylum tricornutum & Ptri \\
\hline & & Thalassiosira pseudonana & Tpse \\
\hline Haptophyta & & Emiliania huxleyi & Ehux \\
\hline Cryptophyceae & & Guillardia theta & Gthe \\
\hline
\end{tabular}

\footnotetext{
a Classification at higher ranks is according to Adl et al. (2012)
}

${ }^{\mathrm{b}}$ Internal species codes used for computational analysis 
phospholipid:sterol acyltransferase (PSAT) involved in sterol ester synthesis [41], whereas LCAT1, LCAT3 and LCAT4 mainly participate in phospholipid catabolism [42].

For a proper characterization of the TAG pathway, it is fundamental to distinguish between PDAT and LCAT activities, since most LCATs biochemically characterized are not active with neutral lipids. With that purpose, we generated an LCAT Hidden Markov Model (called profile $\mathrm{hmm}$ ) that would allow for the identification of all the members of the superfamily, while using subsequent phylogenetic and clustering analyses to classify the superfamily members to each one of the groups. Figure 1 shows a phylogentetic tree of PDATs and LCATs from algae and other representative taxa. Table 2 summarizes the number of PDATs identified in algae, while Additional file 2 shows a detail of all the LCAT superfamily homologs identified. Among green algae, Chlorophyceae have one PDAT and one LCAT1 homolog, whereas Mamiellophyceae do not appear to have LCATs. LCAT2 homologs seem to be unique to SAR algae. In fact, diatoms show LCAT2 and no LCAT1 homologs. PDAT and LCAT2 appear to have evolved from a most recent common ancestor, evidenced by the statistical support of the bifurcation (ML bootstrap value $=100)$.

It was reported previously that human LCAT contains several structurally conserved elements [43, 44], including a catalytic triad of Ser-181-His-377-Asp-345, a salt bridge between Asp-145 and Arg-147, and a so-called lid region. These features, as well as the conserved amino acids, were also identified in plant PDATs [33]. For data mining, we used these conserved motifs to scrutinize sequences and correct MSAs. Figure 2 shows that most of the sequences identified have all the motifs, whereas a few are truncated in either the amino or carboxy end. Since gaps in MSAs are considered ambiguity, some of these last sequences were not used for phylogenetic tree reconstruction. In these cases, clustering within the PDAT or LCAT group was determined by examining the scores obtained when the sequences were confronted with group-specific profile hmms. The MSA in Fig. 2 shows the conserved motifs that are most important for activity in all algae PDATs, as well as others from representative taxa.

\section{DGAT1 in-silico analyses}

DGAT1 is part of a clan of proteins named MBOAT (for membrane bound O-acyl transferase) that contains a variety of membrane acyltransferase enzymes. One of the superfamilies within the MBOAT clan is the sterol Oacyltransferase (SOAT) group (Interpro family IPR014371), which in fact contains three different families. The first one is the SOAT/ACAT family, whose best characterized members are mammalian SOAT1 and SOAT2 (also known as ACAT1 and ACAT2), which play a role in the formation of fatty acid-cholesterol esters [45]. The second is the ARE family (for acyl-coenzyme A: cholesterol acyl transferaserelated enzyme), which contains the fungal homologs of animal SOATs [46]. The third family is the diacylglycerol O-acyltransferase 1 (DGAT1) family.

Figure 3 shows a phylogentic tree of the SOAT/ARE/ DGAT superfamily. The SOAT clade contains all the SOATs from animals, and a related subclade of SOATlike proteins from ciliate protists. The ARE clade contains the previously characterized enzymes from yeast and other fungi, as well as several proteins from nonphotosynthetic protists and from red algae. Last, the DGAT1 clade contains members from most algae analyzed, as well as homologous proteins from many related and unrelated organisms within the tree of life. Amongst green algae, all chloropycean and trebouxiophycean species analyzed show a single DGAT1, whereas mamiellophycean species appear not to have DGAT1 (Table 2). From SAR algae, only Symbiodinium minutum (Alveolata), Bigellowiella natans (Rhizaria) and the diatom Pseudo-nitzschia multiseries appear not to have DGAT1. Interestingly, there is a fourth clade in the superfamily constituted exclusively of proteins from red algae and glaucophyta and from the heterolobosean Naegleria gruberi (Fig. 3). When confronting the proteins within this clade to DGAT1-, ARE- and SOAT -specific profile hmms, all the members of this group show very similar scores to both ARE and DGAT1 proteins (data not shown). Considering that red algae have ARE homologs, these proteins could be DGAT1 proteins that have diverged significantly from conventional DGAT1s. Hence, we termed this the DGAT1-like Clade. Indeed, phylogenetic analysis shows that the DGAT and DGAT1-like clades are related by a most recent common ancestor (ML bootstrap support $=100$ ), further supporting the idea that the members of the DGAT1-like clade might have DGAT1 activity. The main differences in the conserved amino acids, as described by Cao et al. [29] between this clade and the DGAT1 group lie within motifs 5 and 7 (Fig. 4). Some of the modifications seem to be unique to this clade, such as a conserved proline $(\mathrm{P})$ in motif 5 of DGAT1s that is replaced with a glutamic acid (E) only in the DGAT1-like clade. Other differences are shared by other non-algal proteins, mainly metazoans, such as two phenylalanine (F) replacements to methionine $(\mathrm{M})$, leucine $(\mathrm{L})$ or valine $(\mathrm{V})$ in motif 7.

\section{DGAT2 in-silico analyses}

DGAT2 is represented by Pfam DAGAT family. It belongs to the Pfam clan named Acyltransferases (CL0228), which also contains glycerol-3-phosphate acyltransferase (GPAT), acylglycerol-3-phosphate acyltransferase (AGPAT) and other protein families with whom DGAT2 has a modest similarity. DGAT2 is by far the most abundant DGAT in 


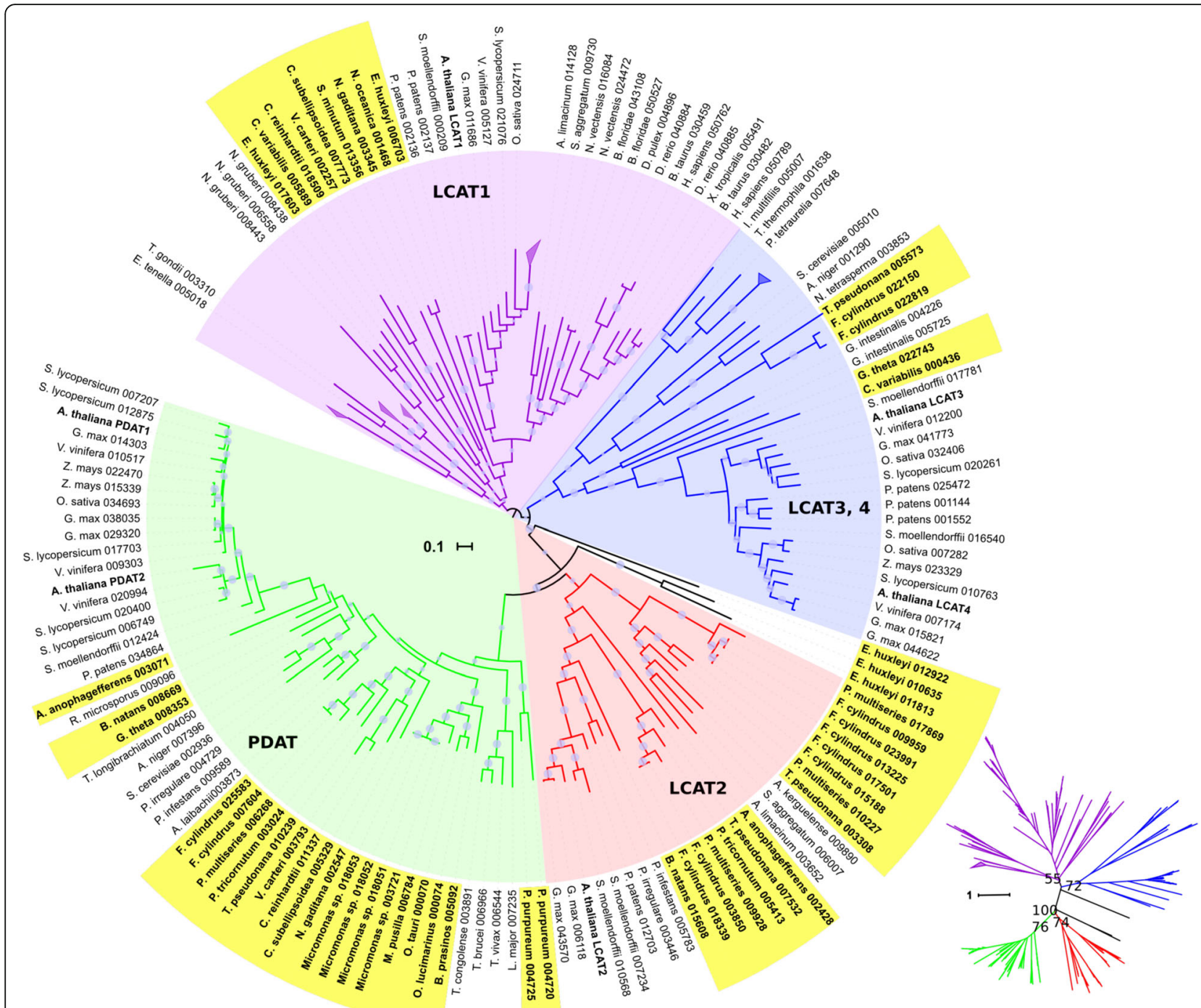

Fig. 1 Phylogenetic relationships of algae members of the LCAT superfamily. Rooted circular phylogram representation of the consensus tree generated by the maximum likelihood (ML) method (500 bootstraps) on the conserved regions of proteins from the LCAT superfamily of the species detailed in Table 1 and Additional file 1. Gray circles represent ML bootstrap values $>50$. Labels highlighted in yellow are algal sequences, numbers after the abbreviated species names are internal IDs. A. thaliana proteins are shown in bold for reference. The scale bar represents 0.1 amino acid substitution per site. A few clades were collapsed for simplicity. The inset is the same tree as an unrooted radial phylogram, with the ML bootstrap values for the four main clades: PDAT, LCAT1, LCAT2 and LCAT3-4. For the inset, the scale bar represents 1 amino acid substitution per site. To view the trimmed MSA used to reconstruct the tree in Phylip format and the raw consensus tree in Newick format, see Additional file 6

algae [34, 47]. The precise number of homologs of each species cannot be unambiguously determined, since many of the sequences identified using sensitive data-mining are almost identical to each other, complicated by the fact that many genomes do not have extensive curation and have several examples of redundant predicted proteins coming from the same gene model. Many of the sequences identified for DGAT2 contain small replacements in conserved residues that are shared among a group of proteins, raising the question of whether those replacements might be functional. Others are truncated and cannot be used for phylogenetic tree reconstruction. Since the similarity between
DGAT2 and the rest of the acyltransferase clan is indeed limited, we did not analyze the phylogenetic relationships of the superfamily. In this case, we retrieved the sequences from algae that had all the conserved motifs (Table 2 and Additional file 3), as described by Cao et al. [29], even if they had small replacements. For instance, we identified six DGAT2s in C. reinhardtii (Fig. 5a), while historically there had been five homologs reported (DGTT1-DGTT5). Two of them have all the completely conserved residues (DGTT2 and DGTT4), while the rest show replacements at different residues of motif five $(\mathrm{V})$, the so-called VPFG block. DGTT5 and Cre08.g377300 have an F to I or to M 
Table 2 Number and predicted chloroplast localization ${ }^{\mathrm{a}}$ of DGAT1, DGAT2, DGAT3 and PDAT sequences identified in algae ${ }^{\mathrm{b}}$ by HMMER iterative data mining

\begin{tabular}{|c|c|c|c|c|c|c|c|}
\hline \multicolumn{4}{|l|}{ Algae } & \multirow{2}{*}{$\frac{\text { DGAT1 }}{1^{c}}$} & \multirow{2}{*}{$\frac{\text { DGAT2 }^{\mathrm{e}}}{1}$} & \multirow{2}{*}{$\begin{array}{l}\text { DGAT3 } \\
-\end{array}$} & \multirow{2}{*}{$\frac{\text { PDAT1, } 2}{1}$} \\
\hline Archaeplastida & Glaucophyta & & Cyanophora paradoxa & & & & \\
\hline & Red Algae & Cyanidiophyceae & Cyanidioschyzon merolae & $1^{c}$ & 1 & - & - \\
\hline & & & Galdiera sulphuraria & $1^{c}$ & 1 & - & - \\
\hline & & Florideophyceae & Chondrus crispus & $3^{c, d}$ & - & - & - \\
\hline & & Porphyridiophyceae & Porphyridium purpureum & $1^{\mathrm{c}}$ & 1 & - & 2 \\
\hline & Green Algae & Trebouxiophyceae & Coccomyxa subellipsoidea & 1 & 4 & 1 & 1 \\
\hline & & & Chlorella variabilis & 1 & 4 & $1-\mathrm{CH}$ & - \\
\hline & & Chlorophyceae & Chlamydomonas reinhardtii & $1-\mathrm{CH}$ & 6 & $1-\mathrm{CH}$ & $1-\mathrm{CH}$ \\
\hline & & & Volvox carteri $f$. nagariensis & 1 & 4 & $1-\mathrm{CH}$ & 1 \\
\hline & & Mamiellophyceae & Bathycoccus prasinos & - & $3-\mathrm{CH}$ & $1-\mathrm{CH}$ & $1-\mathrm{CH}$ \\
\hline & & & Micromonas pusilla & - & $4-\mathrm{CH}$ & $1-\mathrm{CH}$ & $1-\mathrm{CH}$ \\
\hline & & & Micromonas sp. RCC299 & - & 6 & $1-\mathrm{CH}$ & $4^{d}$ \\
\hline & & & Ostreococcus lucimarinus & - & 4 & $1-\mathrm{CH}$ & 1 \\
\hline & & & Ostreococcus tauri & - & $4-\mathrm{CH}$ & 1 & 1 \\
\hline \multirow[t]{10}{*}{ SAR } & Rhizaria & & Bigelowiella natans & - & 6 & - & 1 \\
\hline & Alveolata & Dinoflagellata & Symbiodinium minutum & - & 17 & - & 1 \\
\hline & Stramenopiles & Eustigmatales & Nannochloropsis gaditana & 1 & 8 & - & 1 \\
\hline & & & Nannochloropsis oceanica & 1 & 11 & - & 1 \\
\hline & & Pelagophyceae & Aureococcus anophagefferens & 1 & 5 & - & 2 \\
\hline & & Phaeophyceae & Ectocarpus siliculosus & 1 & 6 & - & - \\
\hline & & Diatoms & Fragilariopsis cylindrus & $2^{d}$ & 4 & - & 2 \\
\hline & & & Pseudo-nitzschia multiseries & - & 4 & - & 1 \\
\hline & & & Phaeodactylum tricornutum & 1 & 4 & - & 1 \\
\hline & & & Thalassiosira pseudonana & 1 & 3 & - & 1 \\
\hline Haptophyta & & & Emiliania huxleyi & 1 & 7 & - & 1 \\
\hline Cryptophyceae & & & Guillardia theta & 1 & 3 & - & 1 \\
\hline
\end{tabular}

${ }^{a}$ Sequences with predicted chloroplast targeting are indicated with bold, italicized numbers followed by $\mathrm{CH}$. ${ }^{\mathrm{b}}$ SAR, Stramenopiles-Alveolata-Rhizaria. ${ }^{\mathrm{c}}$ DGAT1-like clade. ${ }^{d}$ These proteins are very similar to each other and correspond to gene models coming from the same contig region. They are most likely one protein. ${ }^{e}$ For DGAT2, only full-length proteins are reported here

modification, DGTT3 has a $\mathrm{P}$ to $\mathrm{C}$ replacement and DGTT1 has an F to Y modification. It is noteworthy that DGTT1 (CrDGAT2B) has biochemical evidence [9, 48], which indicates that the $\mathrm{F}$ to $\mathrm{Y}$ replacement results in an active protein. Hence, so far we cannot discard sequences that show small differences in the "completely conserved" residues, since amino acid replacement may not necessarily result in a loss of activity $[9,49]$.

Phylogenetic analysis of all the full-length DGAT2s from algae and other eukaryotic taxa uncovered interesting facts. With the exception of a few well-supported orphans, DGAT2 proteins cluster in four main groups (Fig. 6). All the animal and fungal DGAT2 sequences cluster together, in what we called Clade I or the animal-type DGAT2 clade. All the algae higher ranks have sequences related to this clade, albeit in different subclades. The second group contains all the DGAT2 sequences from plants, and numerous members from most of the algae analyzed. We called this Clade II or the plant-type clade. Interestingly, this clade only contains sequences from photosynthetic eukaryotes, regardless of their taxonomic classification. The third clade consists exclusively of protein sequences from photosynthetic and non-photosynthetic SAR species, with the exception of two sequences from the haptophycean alga Emiliania huxleyi. Hence, we called this Clade III or the SAR-type DGAT2 group. Finally, the last clade contains several sequences from SAR, single members from most green algae and a few sequences from Excavata. We called this Clade IV. A sequence logo analysis of motifs 1-6 shows that, with the exception of a few residues, all the clades show a high level of conservation in the completely conserved amino acids (Fig. 5b). Regarding $C$. reinhardtii DGAT2, DGTT1 clusters in the animal-type clade, whereas DGTT2, DGTT3, DGTT4, and DGTT5 


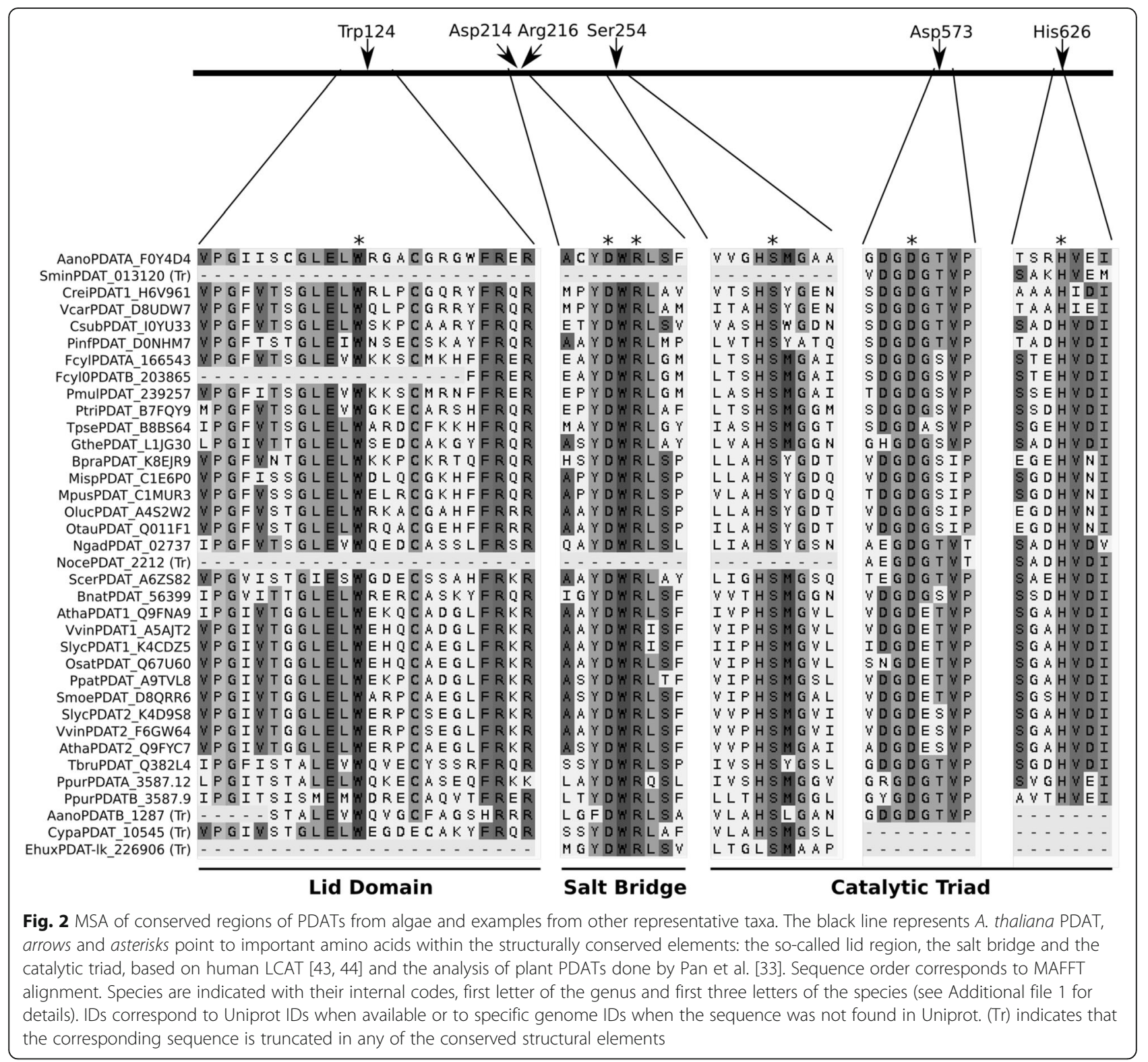

cluster in the plant-type clade, although in different subclades, similarly to what was previously reported [48]. The newly identified DGAT2, corresponding to gene ID Cre08.g377300, clusters in Clade IV. A majority of the proteins within this clade share the residue replacements present in the $C$. reinhardtii homolog (Fig. 5b). Most interestingly, a majority of the members of this clade contain a strikingly high number of predicted transmembrane domains (e.g. six for C. reinhardtii, seven for B. prasinos, nine for $C$. variabilis, eight for $V$. carteri), as opposed to the remaining three clades, which contain an average of two or three transmembrane segments, with very few sequences above this number (Additional file 4). This could point to important differences, worth exploring, in DGAT2-membrane association and catalysis.

\section{DGAT3 in-silico analyses}

One of the most remarkable findings of our data mining was the identification in green algae of sequences with moderate similarity to a soluble type of DGAT, DGAT3 (Table 2), to this date only characterized in a handful of higher plants, including $A$. thaliana [24], tung tree (Vernicia fordii) [40] and peanut (Arachis hypogaea) [39]. DGAT3 proteins are composed of an aminoterminal region containing several disordered stretches and a carboxy-terminal thioredoxin-like $2 \mathrm{Fe}-2 \mathrm{~S}$ clusterbinding domain (2Fe-2S hereafter). When performing HMMER data mining starting with a profile hmm file containing all well-characterized plant DGAT3 proteins, many of the sequences identified with high scores were similar to DGAT3 only in their $2 \mathrm{Fe}-2 \mathrm{~S}$ domain. The 


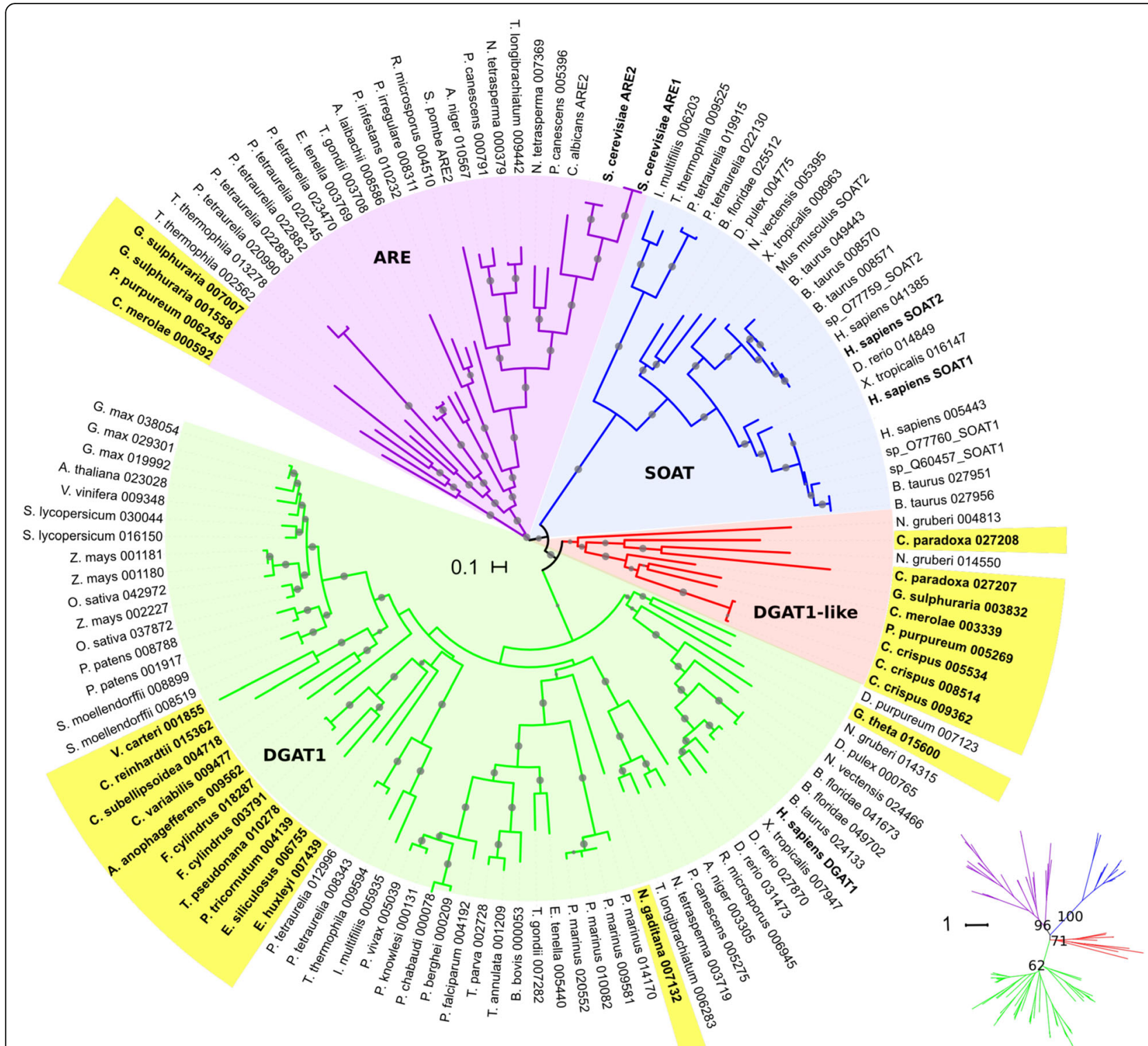

Fig. 3 Phylogenetic relationships of algae members of the MBOAT superfamily. Rooted circular phylogram representation of the tree generated by the maximum likelihood (ML) method (500 bootstraps) on the conserved regions of proteins from the MBOAT superfamily of the species detailed in Table 1 and Additional file 1. Gray circles represent ML bootstrap values > 50. Labels highlighted in yellow are algal sequences, numbers after the abbreviated species names are internal IDs. Homo sapiens and Saccharomyces cerevisiae proteins are shown in bold for reference. Other SOAT and ARE proteins from species not included in Additional file 1 were also added to the analysis and are shown by their Uniprot IDs. The scale bar represents 0.1 amino acid substitution per site. A few clades were collapsed for simplicity. The inset is the same tree as an unrooted radial phylogram, with the ML bootstrap values for the four main clades: SOAT, ARE, DGAT1 and DGAT1-like. For the inset, the scale bar represents 1 amino acid substitution per site. To view the trimmed MSA used to reconstruct the tree in Phylip format and the raw consensus tree in Newick format, see Additional file 7

disordered amino terminal segments of many of these proteins produced MSAs with considerably high entropy that were not suitable for phylogenetic analysis. This prompted us to do a first phylogenetic reconstruction based on the relationships of the $2 \mathrm{Fe}-2 \mathrm{~S}$ domain, which in pfam is known as the 2Fe-2S_thiored domain. The resulting ML tree (inset in Fig. 7) shows two main wellsupported groups. The first major group consists of several subclades of the $24-\mathrm{kDa}$ subunit of respiratory complex I
NADH:ubiquinone oxidoreductase from both prokaryotes and eukaryotes (nuoE gene in Escherichia coli, ndufv2 gene in humans). We refer to this clade as the Nuo24 clade. The other major group (shown in detail in Fig. 7) can be divided into several statistically supported clades of sequences with distinct putative catalytic activities: i) cyanobacterial $2 \mathrm{Fe}-2 \mathrm{~S}$ thioredoxin-like ferredoxins, ii) bacterial and archaean CbiX proteins, a family of cobalt-chelatases that function in the anaerobic biosynthesis of cobalamin (vitamin B12) [50] 


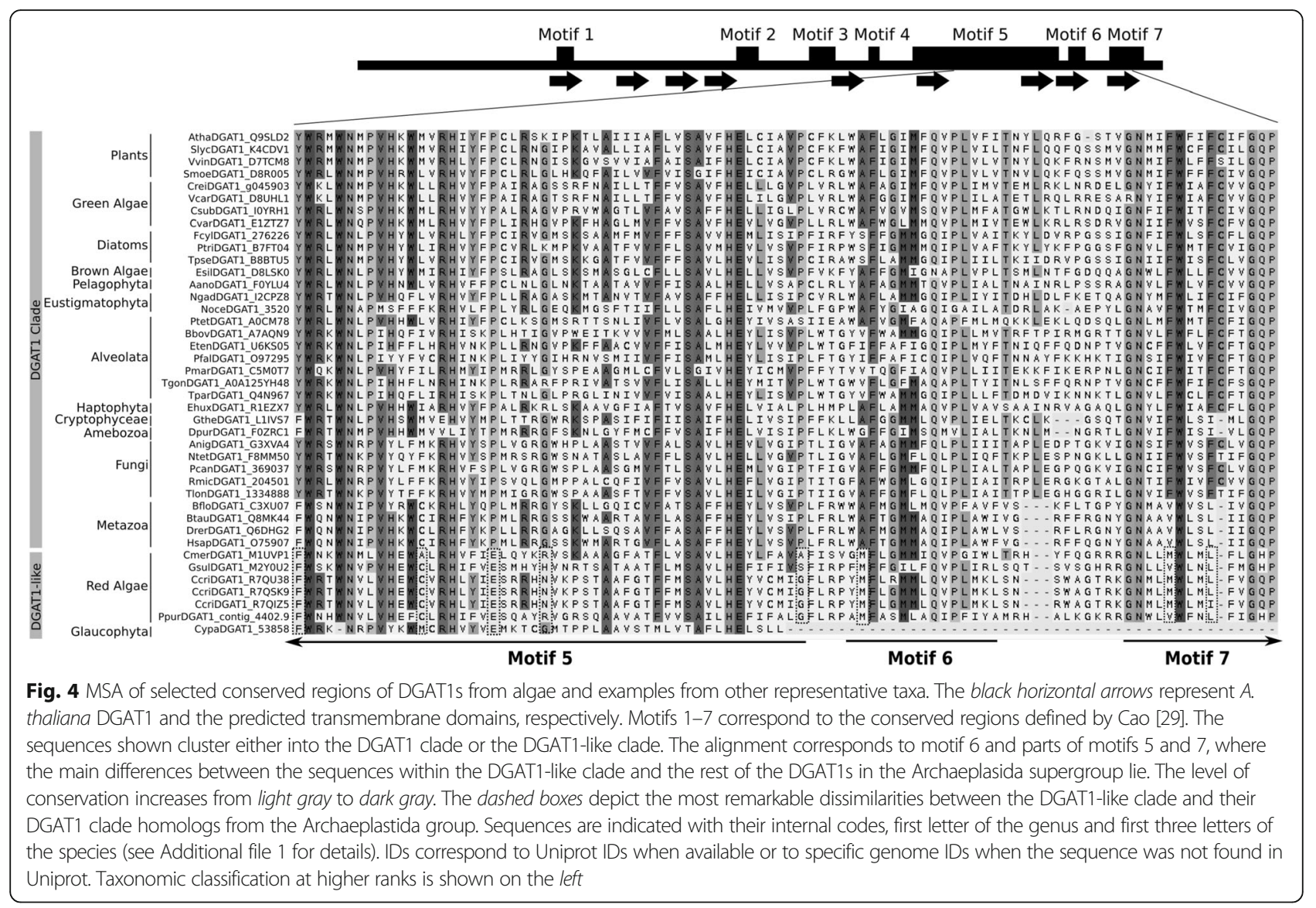

and a group of related uncharacterized proteins from plants (streptophytes); iii) bacterial sucrases, proteins that resemble ferredoxin and appear to have sucrolytic activity [51]; iv) a group composed of both bacterial Nuo51 proteins (the 51-kDa subunit of respiratory complex I NADH:ubiquinone oxidoreductase) and several cyanobacterial hoxF proteins (a subunit within the diaphorase moiety of the reducing hydrogenase) [52] and v) a group of bacterial proteins distantly related to Nuo24.

The last monophyletic clade in Fig. 7 with moderate support (ML bootstrap support $=64$ ) is composed of two subgroups. The first one comprises the thioredoxin-like $2 \mathrm{Fe}-2 \mathrm{~S}$ domains from a group of uncharacterized proteins from cyanobacteria. Although some of the homologs within this group are reported as nucleic acid-binding proteins, this is mostly based on the presence of an OB fold, which might be involved in the binding to other molecules. The second group includes thioredoxin-like $2 \mathrm{Fe}-2 \mathrm{~S}$ domains present in well-characterized DGAT3 proteins, such as the $A$. thaliana (Uniprot ID: Q9C5W0_ARATH) and A. hypogaea DGAT3 homologs (A8VTA4_ARAHY), as well as putative DGAT3 proteins from all the green algae included in the analysis. Interestingly, the DGAT3 group is composed exclusively of 2Fe-2S from Chloroplastida (plants and green algae), with no homologs identified in red algae or glaucophytes. The only exception was the clustering within this group of a thioredoxin-like $2 \mathrm{Fe}-2 \mathrm{~S}$ domain of a protein from the Deltaproteobacteria Haliangium ochraceum. This domain was the only one within the DGAT3 clade that obtained a lower score when confronted to the DGAT3-specific hmm file than to the hmm files of several other clades within the tree. This, together with the long branch length of the corresponding leaf and the heterogeneity of its positioning when analyzing many of the compatible trees used by PhyML to build the consensus (data not shown), puts a question mark on the proper clustering of this sequence.

When eliminating sequences that contributed to the high entropy of the full DGAT3 MSAs, we could establish phylogenetic relationships of DGAT3 sequences using a much larger portion of the proteins, which included most of the amino end. The resulting tree, shown in Fig. 8, shows that the DGAT3 clade is only composed of proteins from higher plants and green algae, with excellent support provided by both ML and bayesian analyses. In addition, this tree supports our findings that reveal that the DGAT3 group has a most recent common ancestor with a group of cyanobacterial $2 \mathrm{Fe}-2 \mathrm{~S}$ proteins.

Since algal DGAT3 proteins have not been previously characterized, we decided to do a series of in-silico and 


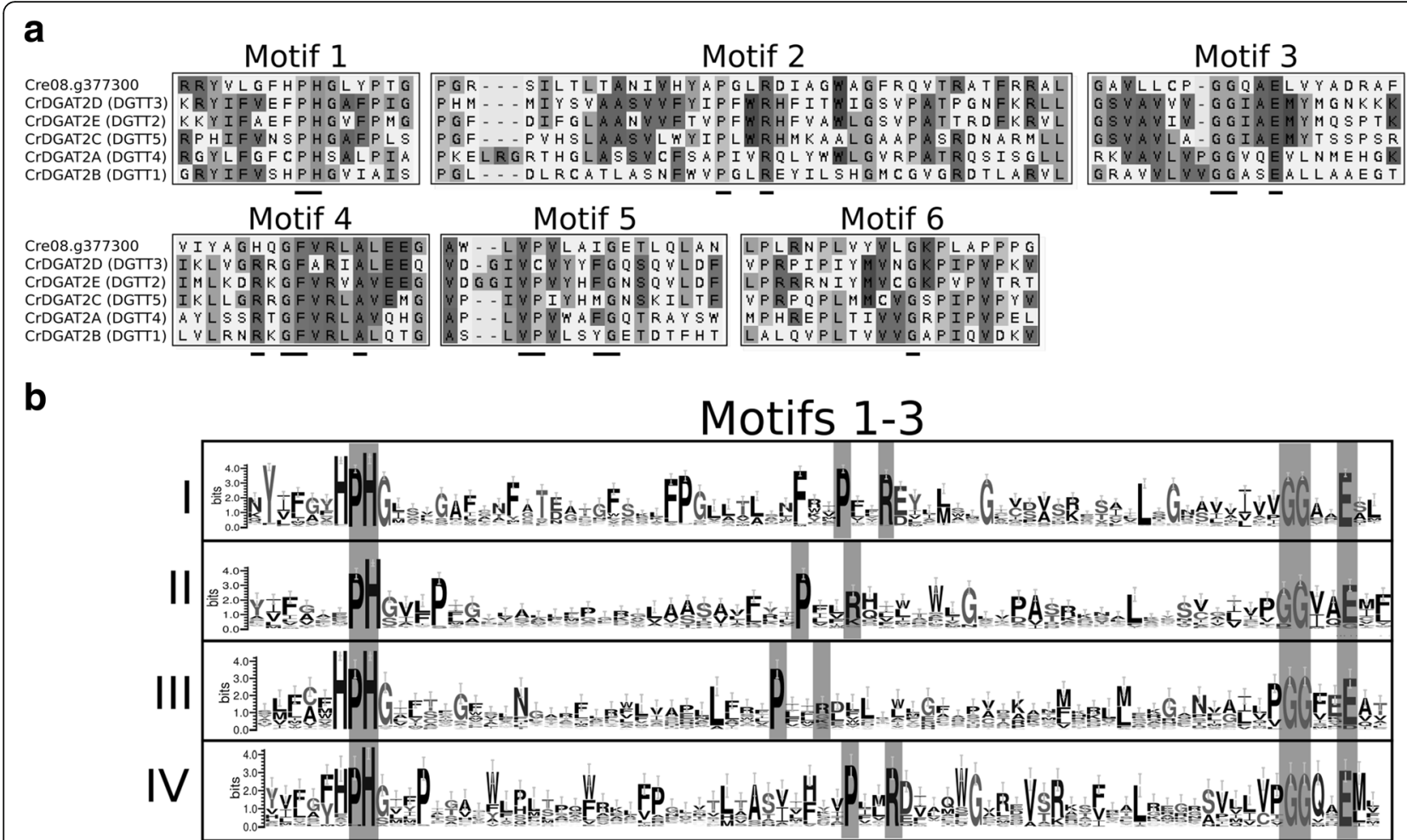

Motifs 4-6

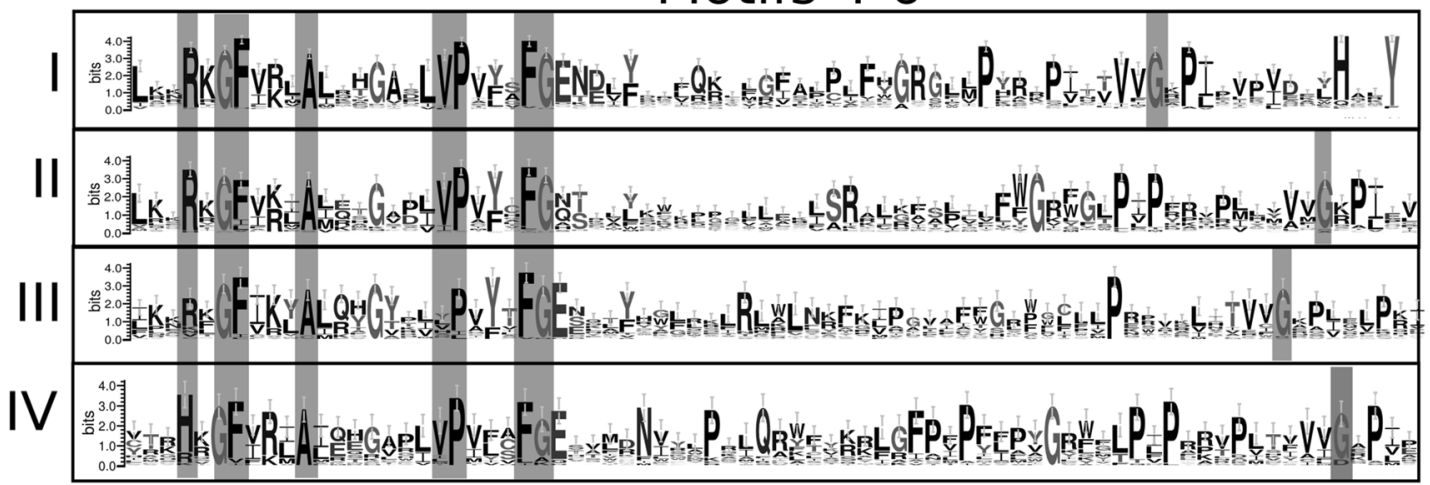

Fig. 5 Sequence features of algal DGAT2 proteins. a MSA of the six C. reinhardtii DGAT2 identified by data mining. The six conserved motifs are shown. The level of residue conservation increases from light gray to dark gray. Underlined amino acids correspond to the completely conserved residues defined by Cao [29]. b Sequence logos of motifs 1-6 for the four main clades (I-IV) of the DGAT2 family. The $y$ axes show the logo bits; error bars are shown on each position. Completely conserved residues are highlighted

empirical analyses with the purpose of obtaining further information about this emerging protein family. Figure $9 \mathrm{a}$ shows the amino acid sequence of $C$. reihardtii DGAT3. The carboxy-terminal region features the four cysteines involved in $2 \mathrm{Fe}-2 \mathrm{~S}$ binding, as well as amino acids involved in dimerization of the polypeptide, as determined by amino acid similarity using delta-blast. Figure 9 a also shows the 91 amino acids that could be modeled with a $99.8 \%$ confidence on the thioredoxin-like 2Fe-2S ferredoxin from Aquifex aeolicus (template d1f37b). In contrast, the formation of dimers could not be modeled with acceptable confidence by
I-COTH, from the I-Tasser package. Secondary structure analysis predicted that $C$. reinhardtii DGAT3 is composed of $40 \%$ disordered regions, $47 \%$ of alpha helices and $9 \%$ of beta strands. Disordered regions are mainly concentrated at the amino terminus of the protein. The catalytic site of DGAT3 is not known for any of the members identified so far. However, the catalytic residues of many acytransferases have been determined, and a histidine $(\mathrm{H})$ seems to be mandatory for activity in most of the protein families, often accompanied by an aspartic (D) or glutamic acid (E) separated by a few amino acids, most frequently four. In the 


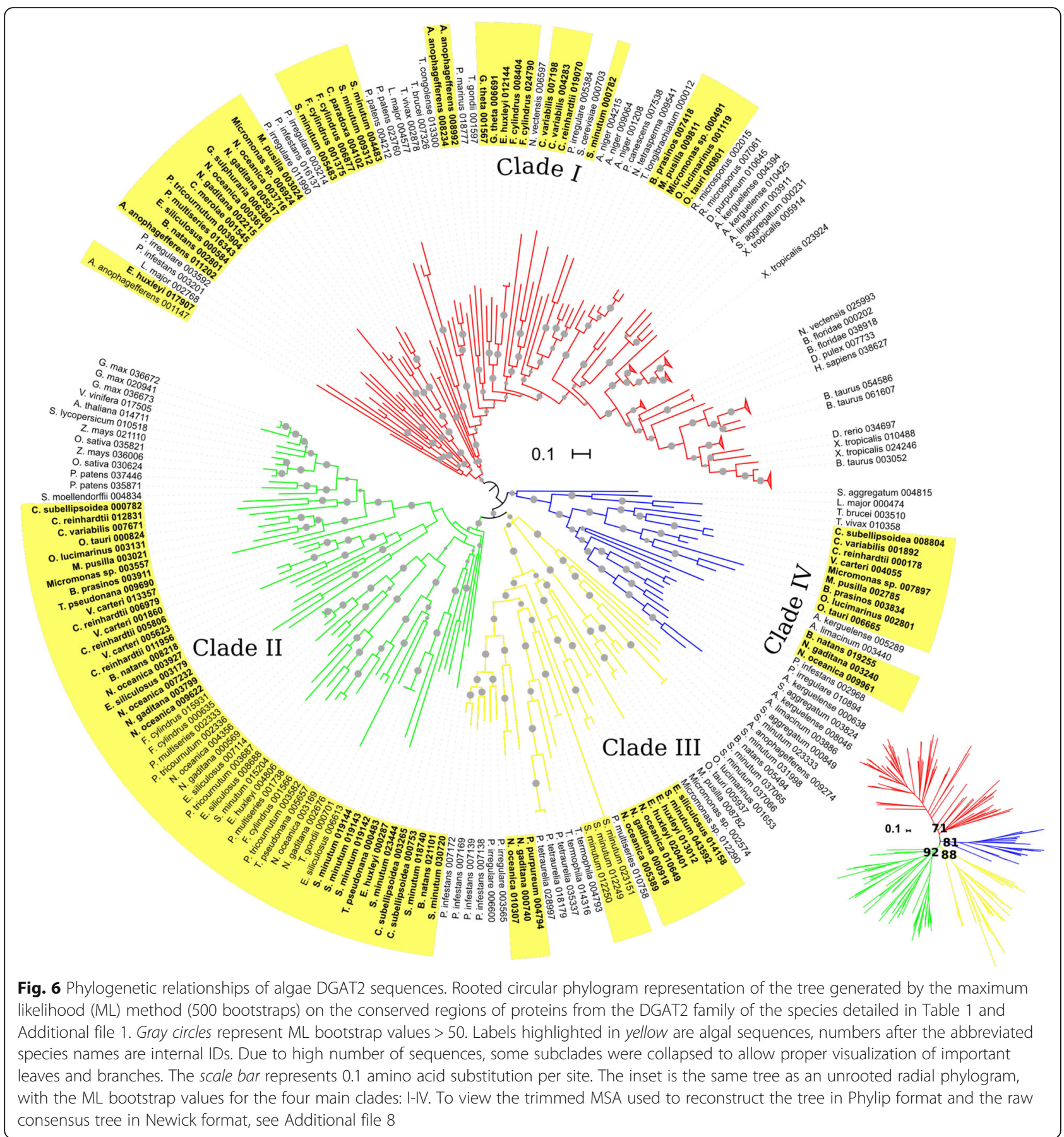

DGAT1 and DGAT2 families, this motif is towards the carboxy end of the protein $[29,30]$, whereas it tends to be towards the middle portion of the protein or the amino end in GPAT and AGPAT [53]. We identified three motifs in $C$. reinhardtii DGAT3 that are similar to acyltransferase motifs, shown in Fig. 9a. Two of them are in the aminoterminal portion, whereas the other one is to the carboxy terminus of the protein, further to the carboxy end than the $2 \mathrm{Fe}-2 \mathrm{~S}$ domain. It is noteworthy that the histidine in the motif closest to the amino end is conserved within the whole DGAT3 clade, as shown in the sequence logo in Fig. 9b.

Figure 9c and Additional file 5 show a hydrophobicity and transmembrane segment comparison between $C$. reinhardtii DGAT1, DGTT4 (a functionally characterized DGAT2,Cre03.g205050) and DGAT3. The three sequences showed a considerable percentage of hydrophobic regions (45, 40 and 25\% for DGAT1, DGAT2 and DGAT3, respectively). Interestingly, in DGAT1 many of its hydrophobic regions translate into transmembrane segments, while only two 


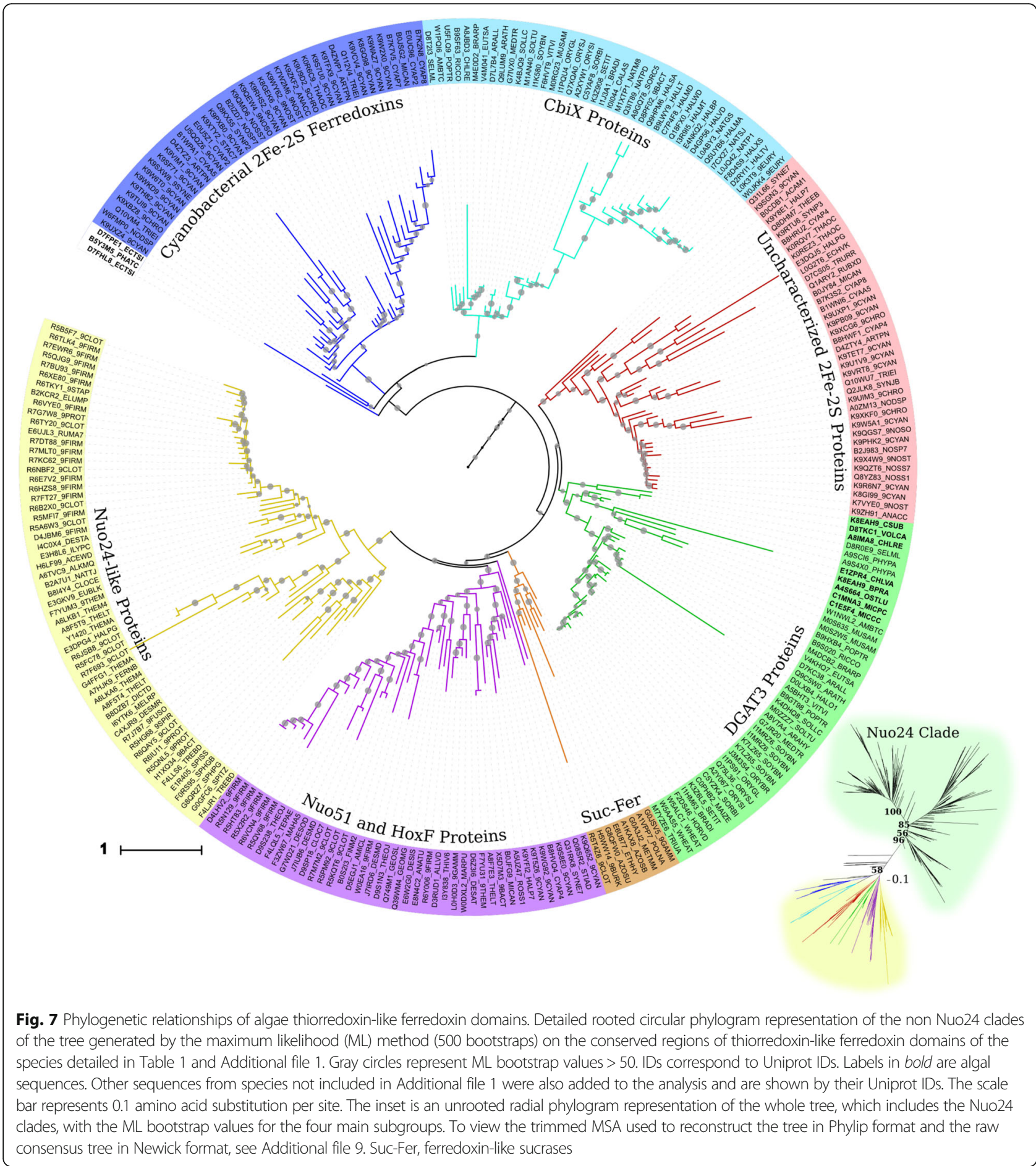

hydrophobic regions represent transmembrane segments in DGAT2 and none in DGAT3. One of the most remarkable findings is that, in DGAT1 and DGAT2, the catalytic motifs either flank or are partially embedded in hydrophobic regions, while the putative catalytic motifs of $C$. reinhardtii DGAT3 are flanked by hydrophilic regions (Fig. 9c). This observation supports the idea that
DGAT3 likely participates in a soluble TAG pathway. Nevertheless, the presence of hydrophobic regions in C. reinhardtii DGAT3 is indicative of both protein membrane association and the interaction with lipid substrates.

We also did subcellular localization prediction analyses of DGAT3 proteins. Table 2 shows that the DGAT3 


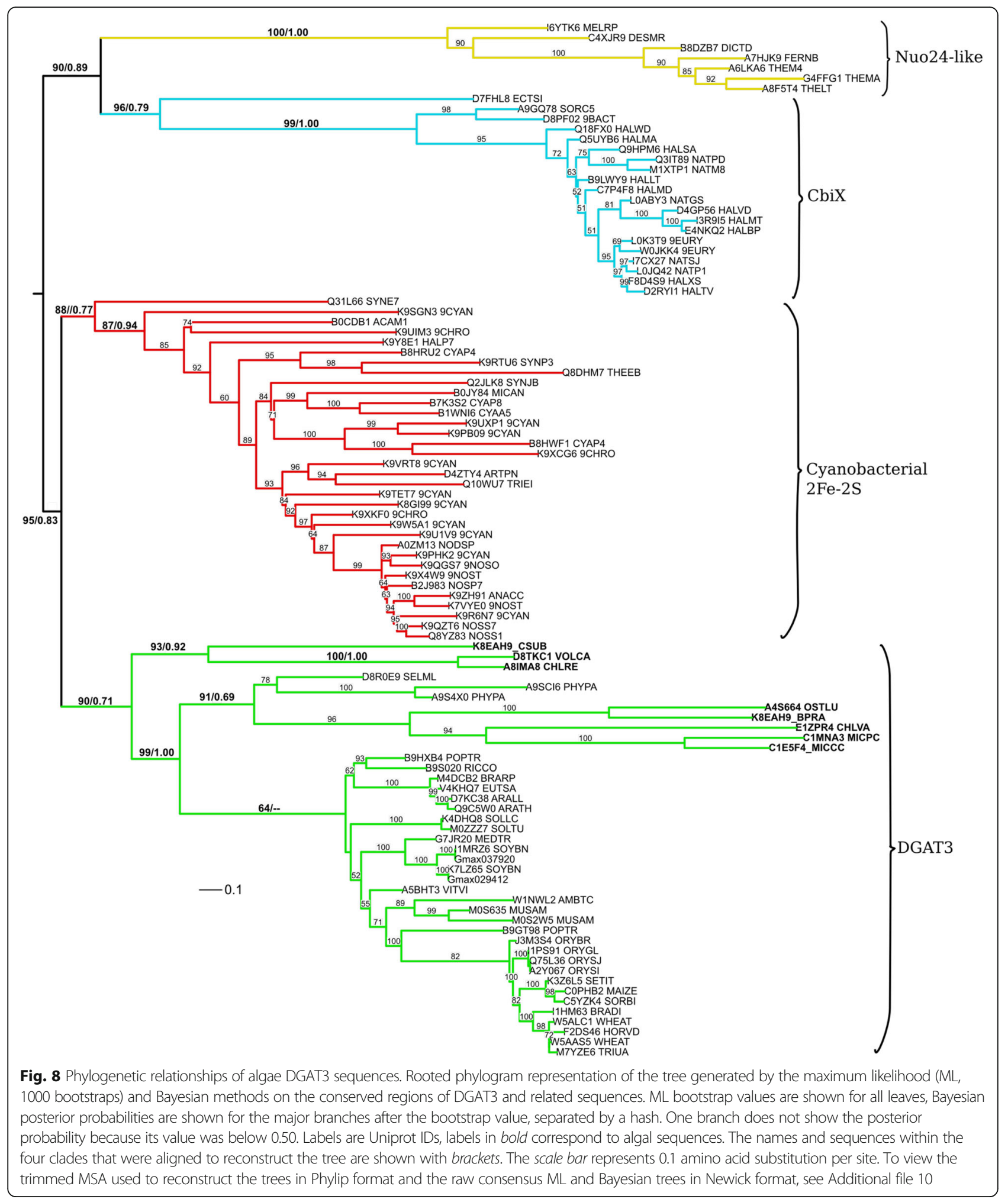

proteins from most of the green algae analyzed, with the exception of the homolog from Coccomyxa subellipsoi$d e a$, were predicted to be localized to the chloroplast using PredAlgo, an algorithm specifically trained with green algal proteins. The Ostreococcus tauri DGAT3 has a truncated amino end due to several unidentified amino acids, which hinders prediction. Although TAGs have been shown to accumulate in the chloroplast under 


\begin{abstract}
a
MASFGLLGRVPTCGELPKCSARSPESLARSGRARAMVPSGFPSVARASHMPTHALVDASRPPAVASPVLC SSLRKERKKLSKVLKSHRKVLERRLSALTTSSVDVDSPLLTELLSELKTLRTSLEGQRSAALLCAADDSS DSDSDDDGCDTRSAAALAAQRRAAATSALTAAASTSQAMTSPARMVVSGLGGQMELTVPELEEGWEW SEADFRAARFEGAPGRVMVCTGSKCQRKGAQQVLEAVSALADGNTNIEVVPCKCVGKCSAGAALRVRP QGQACATYTQVRPAQLRDMFEEHFGAVAAAAGAPSAACCVECKSPGSESQPHLHSHAEGAAVEQHVH QLVA
\end{abstract}

\title{
b

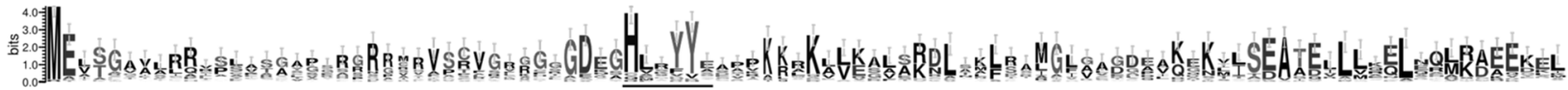 4.0.1.

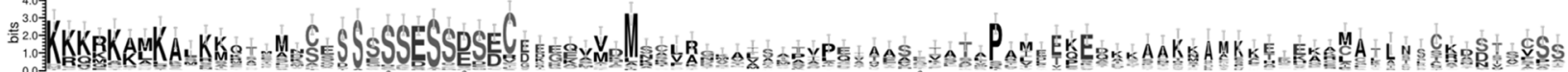

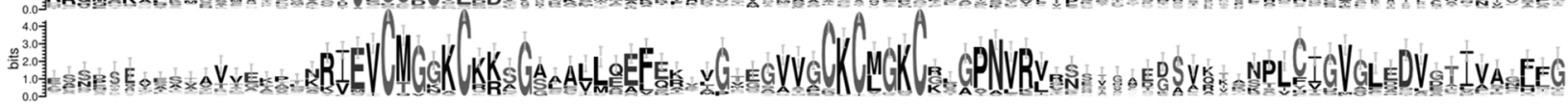 管2.0: \\ C \\ DGAT1 \\ MLGSSPVHTSCRFSLLSSERAWHEKHTGLYNLAFIILVLSNFRLALENALKYGWRLNPVRVVGDLMTGR SGSLGGAFNLPLVACYPLLLAAALLSLGTELAGHALLGQEDKLRQQLSKRSGLSAAAVERAVGLRARAH EWLLFLVHLASTTGVIALPWAVISLTKAEPVSGAILITTAVVLWMKLVSYHHCCFDLRAARRGGEVRPGE RGCPDTPATEWGPLERYPENLTFKNLMYFLAVPTLSYQVNFPRARCVRWRWLLRRCAELCITLTALAILV GQYITPAVDNSLVPLQQLDLPRVAERVLKLALPSTYAWLLGFYCLFHLWLNVLAELTRFGDREFYKDW WNAATVGEYWKLWNMPVHKWLLRHVYFPAIRAGSSRFNAILLTEFVSAVFHELLLGVPLHMVRLWAFA GIMFQVPLIMVTEMLRKKLNRDELGNYIFWIAFCVVG QPVCVLLYYHDYVV
}

\section{DGAT2 (DGTT4)}

MPLAKLRNVVLEYAAIAIYVSAIYTSVVLLPSALALFYLFGATSPSAWLLLAAFLALTFTPLQLTTGALSE RFVQFSVARAAAYFPTRVVVTDPEAFRTDRGYLFGFCPHSALPIALPIAFATTSPLLPKELRGRTHGLASS VCFSAPIVRQLYWWLGVRPATRQSISGLLRARKVAVLVPGGVQ VLNMEHGKEVAYLSSRTGFVRLAVQ HGAPLVPVWAFGQTRAYSWFRPGPPLVPTWLVERISRAAGAVPIGMFGQYGTPMPHREPLTIVVGRPIPV PELAPGQLEPEDEVLAALLKRFTDDLQALYDKHKAQFGKGEELVIM

\section{DGAT3}

MASFGLLGRVPTCGELPKCSARSPESLARSGRARAMVPSGFPSVARASHMPTHALVDASRPPAVASPVL CSSLRKERKKLSKVLKSHRKVLERRLSALTTSSVDVDSPLLTELLSELKTLRTSLEGQRSAALLCAADDS SDSDSDDDGCDTRSAAALAAQRRAAATSALTAAASTSQAMTSPARMVVSGLGGQMELTVPELEEGWE WSEADFRAARFEGAPGRVMVCTGSKCQRKGAQQVLEAVSALADGNTNIEVVPCKCVGKCSAGAALRV RPQGQACATYTQVRPAQLRDMFEEHFGAVAAAAGAPSAACCVECKSPGSESQPHLHSHAEGAAVEQHV HQLVA

Fig. 9 Sequence characteristics of DGAT3. a C. reinhardtii DGAT3 predicted amino acid sequence. Putative catalytic sites are underlined. Highlighted are the cysteines predicted to participate in $2 \mathrm{Fe}-2 \mathrm{~S}$ binding (in gray) and the residues potentially involved in dimerization (in black). The bolded italicized residues correspond to the 91 amino acids modeled on the thioredoxin-like 2Fe-2S ferredoxin from Aquifex aeolicus. b Sequence logo of the DGAT3 family. The sequences within the DGAT3 clade of Fig. 8 were aligned, and the MSA was trimmed using BMGE to eliminate gaps (see Methods). The $y$ axes show the logo bits; error bars are shown on each position. Underlined is the putative acyltransferase catalytic site that is most conserved amongst the members of the clade. c Comparison of C. reinhardtii DGAT1 partial sequence published by Boyle et al. [9], DGAT2 (DGTT4) and DGAT3 sequences. Highlighted in gray are the hydrophobic regions obtained by Kyte \& Dolittle hydrophobicity scale analysis using a window size of 9 . Underlined regions indicate transmembrane segments predicted by TM-HMM analysis. Residues highlighted in black show well established and/or putative conserved catalytic motifs

nitrogen starvation [54, 55] and light stress [56], a de novo pathway for the synthesis of TAGs within this organelle has not been reported yet. In order to extend the results obtained with DGAT3, we did in-silico analysis of protein targeting for all the DGATs and PDATs identified in algae. The results (Table 2) show that some of the DGAT1, DGAT2 and PDAT homologs from green algae are predicted to be targeted to the chloroplast, 
whereas we did not find any cases of predicted chloroplast localization for any of these enzymes in SAR, haptophycean and cryptophycean algae. For C. reinhardtii in particular, we analyzed the complete de novo pathway starting from the activation of fatty acids. Table 3 shows that, with the exception of phosphadidate phosphatase (PAP), all the enzymatic steps of the pathway have at least one isoform with evidence of expression either at the transcriptional or protein level and predicted chloroplast targeting. Our analysis also revealed that this pathway could be soluble, carried out by enzymes associated to chloroplast membranes but with no transmembrane segments (Table 3). This would be consistent with the soluble, albeit cytosolic, pathway proposed in developing cotyledons of peanut, in which DGAT3 is involved [39].

\section{Characterization of $C$. reinhardtii DGAT3 enzymatic activity}

For DGAT families with well characterized members, such as DGAT1 and DGAT2, it is very common to infer activity of a new member by simple similarity analysis. However, DGAT3 is a very incipient family, with very few members characterized. In addition, the DGAT3 family is completely unrelated to DGAT1 and DGAT2, and the conserved sites that are important for activity are not known. With the purpose of interrogating C. reinhardtii DGAT3 catalytic activity, we expressed this protein in E. coli cells (Fig. 10a). Most groups of bacteria, including $E$. coli, do not accumulate TAGs to significant levels, which allows to easily detect the presence of such compounds dependent on the expression of a heterologous enzyme. TLC analysis of $E$. coli total lipids showed that $C$. reinhardtii DGAT3 expression produced an increase in the accumulation of TAGs compared to the control (Fig. 10b). Interestingly, the TAG spot was only visible when gluconate and oleate were added to the culture during induction. The addition of those compounds triggers in the bacterial cell a set of responses collectively known as storage condition, which was previously used with the purpose of driving the bacterial metabolism towards TAG synthesis [57, 58]. The fact that TAGs were not detectable in DGAT3expressing $E$. coli cells without the addition of an excess of substrate (oleate) might indicate that, in a prokaryotic system, DGAT3 activity cannot compete with phospholipid synthesis, which is a major lipid pathway in E. coli. Interestingly, a spot that migrated further than the TAGs appeared in all samples of the TLC. According to its positioning relative to the standards and to results reported by others $[59,60]$, this spot might correspond to quinones, a type of neutral lipid present in E. coli that can be coextracted with TAGs [58]. Future experiments will be aimed at characterizing the DGAT3 activity further and at determining the precise identity of the additional spot in TLCs.

\section{Discussion}

Data-mining and phylogenetic analyses

In this work, we have done a thorough data mining of the TAG pathway, with an emphasis on the phylogenetic connections between homologs of algae from distinct supergroups. We have unveiled interesting relationships of the enzymes involved in the committing steps, DGAT and PDAT, that might have important evolutionary and functional implications. Our data mining and phylogenetic analyses allowed us to make testable predictions on the identity of enzymes that are part of complex superfamilies. Future

Table 3 C. reinhardtii sequences within the TAG pathway with predicted chloroplast targeting

\begin{tabular}{|c|c|c|c|c|}
\hline Phytozome v11.0 ID & Length (aa) & PredAlgo & Membrane (M)/Soluble (S) & Evidence $^{a}$ \\
\hline \multicolumn{5}{|l|}{ LACS } \\
\hline Cre12.g500715.t1.2 & 1242 & $C$ & S & Transcript level \\
\hline Cre06.g299800.t1.2 & 784 & C & M & Transcript level \\
\hline \multicolumn{5}{|l|}{ GPAT } \\
\hline g6130.t1 & 456 & C & M & Protein Level \\
\hline Cre02.g143000.t1.2 & 410 & C & S & Protein Level \\
\hline \multicolumn{5}{|l|}{ GPAT/LPAT } \\
\hline Cre05.g248150.t1.2 & 370 & C & M & Transcript level \\
\hline Cre17.g738350.t1.2 & 325 & C & M & Transcript level \\
\hline \multicolumn{5}{|l|}{ DGAT1 } \\
\hline Cre01.g045903.t1.1 & 464 & C & M & Transcript level \\
\hline \multicolumn{5}{|l|}{ DGAT3 } \\
\hline Cre06.g310200 & 346 & C & S & Transcript level \\
\hline \multicolumn{5}{|l|}{ PDAT } \\
\hline Cre02.g106400 & 1040 & C & M & Protein level \\
\hline
\end{tabular}

\footnotetext{
${ }^{a}$ Transcript level refers to evidence of existence by EST tagging, RNAseq, Microarray or RT-PCR analyses. Protein level refers to evidence by proteomics
} 


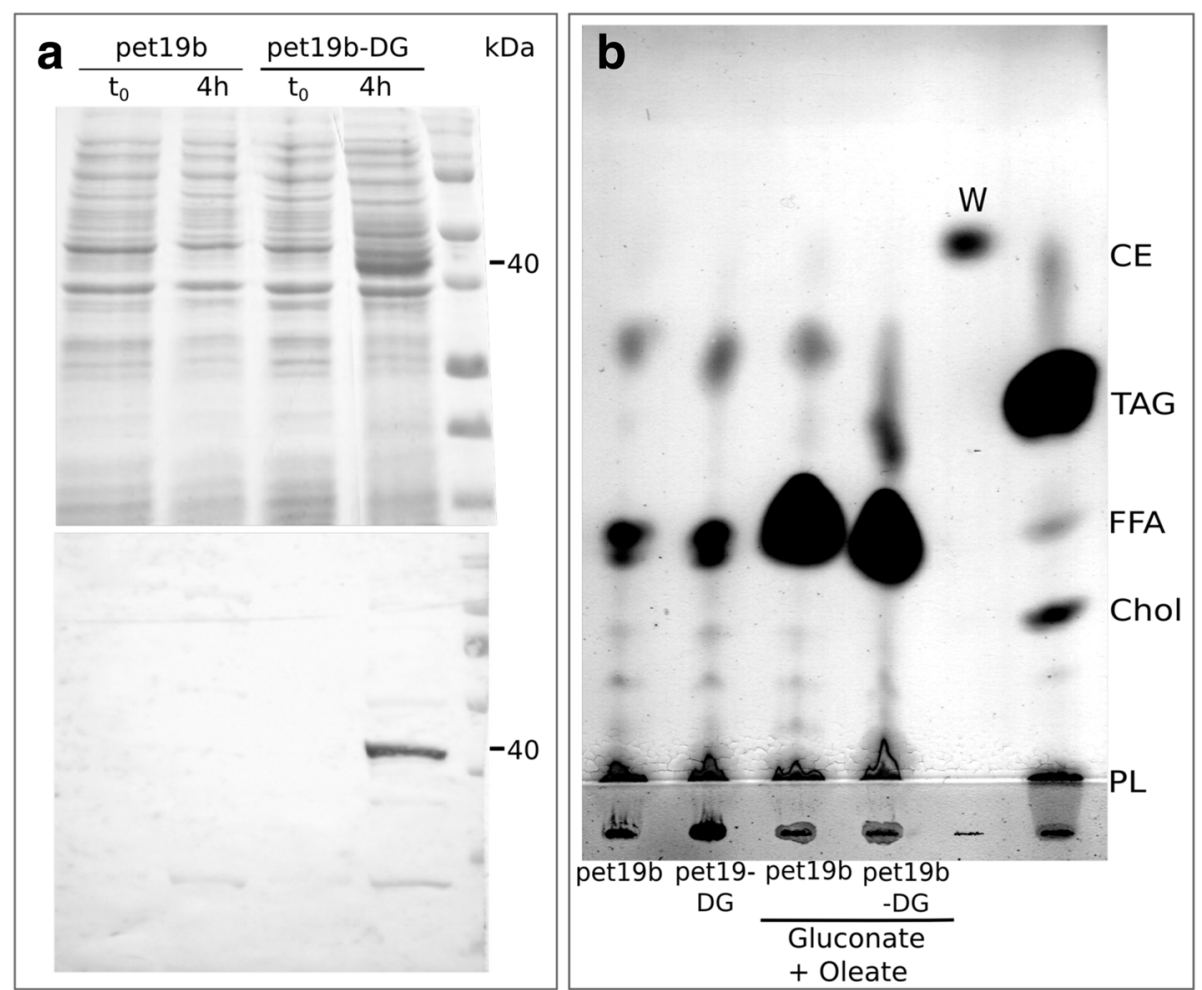

Fig. 10 Characterization of C. reinhardtii DGAT3 expressed in E. coli. The coding sequence of $C$. reinhardtii dgat3 was cloned into pet19b vector (pet19b-DG) and transformed into E coli cells. The empty vector (pet19b) was transformed as a control. Protein induction was done on both DGAT3expressing and control cells. Samples were taken before ( $\left.\mathrm{t}_{0}\right)$ and $4 \mathrm{~h}$ after induction $(4 \mathrm{~h})$. a Soluble proteins from both samples were analyzed by $12 \%$ SDS-PAGE and either stained with Coomassie brilliant blue (upper panel) or transferred to nitrocellulose (bottom pane). The membranes were incubated with rabbit anti-C. reinhardtii DGAT3 polyclonal antisera. The protein standards are shown on the right lanes, the dashes indicate the extra band in the gel upon induction and the protein recognized by the antisera, of approximately $40 \mathrm{kDa}$. b Lipids from E. coli cells were extracted with methanol: chloroform (1:1) and analyzed by thin layer chromatography (TLC) using a mix of hexane-diethyl ether-formic acid as the solvent system. Standards are: $C E$, chloesterol ester; W, wax ester; TAG, triglyceride; FFA, free fatty acids; Chol, cholesterol; PL, phospholipids

experimentation will put to the test the hypotheses arisen from this work.

One of the most remarkable findings of our data mining was the identification of sequences with similarity to plant soluble DGAT3 proteins, exclusively in the green algal lineage. There are several interesting facts about DGAT3. First, phylogenetic analysis of either the $2 \mathrm{Fe}-2 \mathrm{~S}$ domain or a much larger portion of the protein show that the DGAT3 clade shares a most recent ancestor with a group of uncharacterized proteins from cyanobacteria. The highest HMMER search scores of the DGAT3 hmm file were, after the members of the DGAT3 clade, obtained with cyanobacterial proteins. In contrast, no prokaryotic sequences were above the inclusion threshold in the searches done for DGAT1, DGAT2 and PDAT, hence no prokaryotic sequences are included in the phylogenetic trees of these protein superfamilies. These findings suggest that DGAT3 is a very distinctive protein within the TAG-synthesizing pathways. DGAT1, DGAT2 and PDAT are proteins exclusive to eukaryotes, in agreement with the fact that the synthesis of TAGs is widespread in eukaryotic organisms, whereas TAGs accumulate to significant levels only in a few prokaryotic groups [61, 62]. These enzyme superfamilies most likely evolved in eukaryotes coincident with an increase in the complexity of the lipid metabolic pathways, as exemplified by the intricate phylogenetic relationships between DGAT1 and SOAT, or PDAT and LCAT or by the extensive multiplication of DGAT2 in algae. DGAT3, in contrast, is likely a relic of cyanobacterial ancestry.

Plant DGAT3 proteins are reported to be cytosolic, in agreement with their localization prediction. In contrast, most green algal DGAT3 are predicted to be targeted to the chloroplast. We could speculate that the members of Chloroplastida (plants and green algae) inherited DGAT3 from cyanobacteria and, if the localization predictions are confirmed, this protein remained chloroplastic in green algae, whereas it found its eukaryotic functions in the cytosol in higher plants. This would point to another interesting difference between green algae and higher plants regarding TAG regulation. We could further question whether DGAT3 was acquired by the whole 
Archaeplastida supergroup during primary endosymbiosis and subsequently lost in red algae and glaucophytes, or evolved later on only in Chloroplastida. Although the latter seems the most parsimonious explanation, further analyses could certainly allow to differentiate between these two possibilities. Regardless of its origin, DGAT3 seems to be exclusive to the green lineage. Proteins with this characteristic have been clustered within the ViridiCut2 group in a recent inventory of proteins common to plants and green algae [63]. The authors of that analysis determined that most of the ViridiCut2 proteins lack cyanobacterial homologs and might be involved in eukaryotic processes that are not exclusively associated with the photosynthetic function. It is difficult to unambiguously answer whether DGAT3 lacks cyanobacterial homologs, since the function of the members of the cyanobacterial clade related to DGAT3 is not known. Nevertheless, the fact that TAG synthesis is mostly a eukaryotic function that, although related to the plastid, exceeds photosynthesis, suggests that DGAT3 shares this characteristic with other ViridiCut2 proteins.

\section{Characteristics of $C$. reinhardtii DGAT3}

DGAT3 proteins are characterized by the presence of an $2 \mathrm{Fe}-2 \mathrm{~S}$ domain that has a fold similar to that present in thioredoxins [64]. Many of the bacterial proteins containing this domain are small (ca. 80-100 amino acids), soluble lowpotential electron carriers (between -0.2 and $-0.45 \mathrm{~V}$ ) with a single $2 \mathrm{Fe}-2 \mathrm{~S}$ cluster [65]. The exact role of many of these proteins is still unclear. Their homologous domains in larger redox enzymes (such as Nuo24, Nuo51 and NAD-reducing hydrogenases) function as electron carriers [66, 67]. In this scenario, we could imagine that DGAT3 has a dual activity in which electron transfer is coupled to the acylation reaction. Although the precise site for catalytic activity of DGAT3 proteins is not known, there are few important amino acids that seem to be common to many acyltransferases, as analyzed by Saha et al. [39]. We identified three motifs that could catalyze acylation reactions in $C$. reinhardtii DGAT3, two in the amino terminal region and one to the carboxy-terminal portion of the $2 \mathrm{Fe}-2 \mathrm{~S}$ clusterbinding domain. Future experimentation will shed light to the precise site in DGAT3 where catalysis occurs.

\section{The chloroplast TAG pathway and possible involvements of PDAT, DGAT1 and DGAT3}

The importance of a chloroplast pathway for the synthesis of TAGs in green algae became evident with recent work done in C. reinhardtii. Fan et al. [54] showed that, during nitrogen starvation, a high proportion of the TAG produced is composed of 16-carbon (C16) fatty acids in the sn-2 position of the glycerol backbone and is, in fact, mostly synthesized from DAG generated in the chloroplast. The presence of lipid droplets inside the chloroplast was also evidenced by microscopy in starchless $C$. reinhardtii mutants grown in mixotrophic conditions $[54,55,68]$. One major player in the synthesis of chloroplast TAGs in green algae could be PDAT. The $C$. reinhardtii PDAT homolog is known to localize to the chloroplast [69] and to use preferentially C16 fatty acids [70]. A loss-of-function analysis of C. reinhardtii PDAT revealed that it might contribute to approximately $25 \%$ of the TAG accumulation under nitrogen starvation in C. reinhardtii [9]. In contrast, it has been proposed that the contribution of the chloroplast to total TAG synthesis during nitrogen depletion could be much higher [54], suggesting that other mechanisms are involved. Analysis of chloroplast plastoglobuli and cytosolic lipid droplets in the green alga Dunaliella bardawil revealed similar fatty acid composition in the sn- 2 and sn- $1+3$ positions for the TAGs in both types of lipid bodies, suggesting a common origin [71]. Under nitrogen starvation, the formation of cytosolic lipid droplets preceded that of plastoglobuli and appeared to be synthesized via the de novo pathway, whereas that of plastoglobuli seemed to be mostly originated from membrane remodeling [71]. Electron microscopy analysis revealed that the ER membrane and the cytosolic lipid droplets per se attach to the chloroplast envelope, leading the authors to hypothesize that cytosolic TAGs might be transferred to the chloroplast through associations between the ER membrane and the outer membrane of the chloroplast envelope (OMCE) [71]. Such associations were also observed in C. reinhardtii [55]. Therefore, despite the differences in TAG accumulation between the two algae, the idea that interactions between the ER and the OMCE allow the interchange of fatty acids, DAG and TAG between both compartments is likely for all green algae.

In $A$. thaliana, rosette lipid analysis revealed that TAG accumulation increases considerably under certain situations, such as ozone-related stress [72] or senescence [73]. In those conditions, TAGs accumulate preferentially in the chloroplast. Rosette leaf mRNA analysis evidenced that one clone annotated as DGAT1 increased notoriously in senescent leaves compared to young leaves, allowing the authors to propose that DGAT1 has an important role in senescence by sequestering de-sterified fatty acids coming from the thylakoid [73]. The presence of DGAT activity related to plant plastids was shown more than 30 years ago in isolated spinach chloroplasts, where it was found in the chloroplast envelope [74]. Unfortunately, back then, the isoform responsible for activity was not analyzed or identified, but it could be a DGAT1. In this work, localization analysis predicted that C. reinhardtii DGAT1 localizes to the chloroplast. In this scenario, and considering that this protein has transmembrane domains, it is possible that DGAT1 associates not only to the ER, but also to the OMCE, in C. reinhardtii. 
In this context, the participation of DGAT3 could be completely different. Being a soluble, yet membraneassociated, protein with an $2 \mathrm{Fe}-2 \mathrm{~S}$ domain, we could imagine a participation in the stroma, in relationship to the thylakoid membrane. Considering that it might be, the same as its homologous proteins characterized so far, a low potential redox protein, we could hypothesize that $C$. reinhardtii DGAT3 accepts electrons from the final transporters of the photosynthetic electron transport chain and reduces NADP+. The NADPH formed in this way could be used for fatty acid synthesis, which could then be used for TAG synthesis by DGAT3. Furthermore, DGAT3 might be responsible for TAG formation in the chloroplast in situations that produce an excess of electrons. The hypothesis that TAGs might serve as a sink for electrons moving through the photosynthetic electron transport chain has been previously postulated [1]. As previously suggested [75], during stress, excess photosynthetic electrons could be used to generate reducing equivalents, in the form of NADPH, for fatty acid synthesis. Since an excess of free fatty acids is potentially harmful itself, the dual action of DGAT3 would ensure the coupling of NADP+ reduction, fatty acid synthesis and fatty acid acylation into TAG, providing a complete protective mechanism. Although, during stress, much of this could occur in the ER as proposed [75], we hypothesize that the green algal chloroplast might have a complete pathway to do this in an independent fashion.

\section{Conclusions}

To our knowledge this study represents, to date, the most comprehensive sequence analysis of the main enzymes that participate in TAG synthesis in algae, including their phylogenetic relationships with homologs from other organisms and important structure-function predictions. Our results confirmed the complex connections between the distinct acyltransferase superfamilies. Regarding the green alga $C$. reinhardtii, we observed that TAG synthesis is well represented by the presence of numerous acyltransferases, including one PDAT, one DGAT1, six DGAT2 and one DGAT3. DGAT3 is a soluble protein exclusive to the green linage, with predicted chloroplast localization. This protein is the only acyltransferase activity of those analyzed associated to a cyanobacterial heritage. Heterologous expression confirmed that $C$. reinhardtii DGAT3 is indeed a DGAT. As evidenced by subcellular targeting prediction, green algae are likely the only algal group with a chloroplast pathway for TAG synthesis. Methodologically, our analyses allowed us to conclude that data-mining by means of HMMER iterative search followed by phylogenetic analysis and/or protein clustering is a more adequate strategy for lipid functional genome analysis compared to the use of non-iterative heuristic methods, particularly for paraphyletic groups like algae.

\section{Methods}

Proteomes and databases used for data mining

Protein models from fully-annotated genomes (hereafter called complete proteomes, according to http://www.uniprot.org/keywords/KW-0181) [76] of 26 eukaryotic algae groups, supplemented with the complete proteomes of 53 non-algal eukaryotic organisms were used for sequence mining (all available by December 2015). Additional file 1 lists the eukaryotic species analyzed, their internal codes, the source of the proteome sequences and the corresponding references or sequencing projects. We followed taxonomic relationships between taxa according to Adl et al. [13]. Prokaryotic homologs were extracted from Reference Proteomes (EMBL-EBI, http://www.ebi.ac.uk/).

\section{Identification and analysis of algae homologs of the TAG synthesis pathway}

Sequences were identified by HMMER version 3 [77] iterative profiling starting from seed multiple sequence alignments (MSAs). Seed MSAs were constructed with MAFFT at http://mafft.cbrc.jp/alignment/server/index.html [78] or hmmalign [77], using reviewed sequences obtained from Swissprot [76]. Seed MSAs were visualized and manually corrected using Aliview [79] and GeneDoc [80] and subsequently used to generate position-specific scoring tables (Hidden Markov Models, hmm files) using the hmmbuild tool from the HMMER suite. The models generated were used to search a compiled fasta file containing all the complete proteomes of the selected eukaryotic species, using the hmmsearch tool. Sequences above the default inclusion threshold (E-value $=0.01$ ) were retrieved and sequences showing $100 \%$ identity were eliminated using CDHIT [81]. The remaining sequences were aligned using MAFFT. The resulting MSAs were manually corrected. This mainly involved the elimination of sequences that did not have the hallmarks specific for each protein, used for scrutiny, and re-alignment of incorrectly aligned residues. The edited MSAs were used for the generation of new hmm files, re-starting the whole cycle. This process, for each protein, was repeated until convergence, at which point no new information was obtained in a new datamining cycle. In order to ensure that we obtained the most complete datasets possible, the sequences from each phylogenetic group were retrieved and used in group-specific data-mining following the same procedure as the one described above. In order to analyze algae homologs in a true phylogenetic context, the seed and final hmm files were used to search for prokaryotic homologs. This was done by searching (using hmmsearch) the Reference Proteomes databases at the HMMER website, restricted to Bacteria and 
Archaea. All non-identical prokaryotic sequences better than a established inclusion threshold (E-value $=10^{-8}$ ) were added to the analysis.

In order to use only phylogenetically informative regions for the reconstruction of phylogenetic trees, final MSAs were trimmed using BMGE (Block Mapping and Gathering with Entropy) [82]. BMGE optional arguments were determined based on the conservation of secondary structure elements and were: -m BLOSUM30 -h 0.7 -g 0.4:0.2 -b 3. This procedure eliminated not only alignment columns according to entropy and gap content, but also eliminated sequences that contained a gap proportion higher than $40 \%$. All the putative algae sequences that were eliminated using this criteria were confronted with the group-specific hmm profiles mentioned in the previous section, and the closest phylogenetic group was determined by the highest HMMER scores. Sequence logos were generated on desired regions of trimmed alignments using WebLogo 3 (http:// weblogo.threeplusone.com/create.cgi) [83] with default settings and the Chemistry color scheme.

\section{Phylogenetic tree reconstruction}

Maximum Likelihood and Bayesian methods were used for phylogenetic tree reconstruction. For Maximum Likelihood, PhyML 3.0 was used with default settings and 500-1000 bootstraps depending on the MSA [84]. IQ-TREE in ultrafast mode $[85,86]$ with defaults settings was used to obtain a fast consensus ML tree used as a starting tree in bayesian phylogeny reconstruction. For Bayesian analysis, we used Mr. Bayes 3.2.3 [87] with the previously mentioned starting tree with five perturbations and the WAG model, with its own default priors and invariant gamma distribution approximated with four substitutions. Two Metropolis-coupled Markov Chain Monte Carlo (MCMCMC) runs with four chains each and a temperature of $\mathrm{T}=0.05$ were done. Convergence of the chains in the Bayesian analysis was assessed by monitoring that the standard deviation of split frequencies was $<0.01$ and with AWTY online, a system for the graphical exploration of MCMC convergence in Bayesian phylogenetic inference (http://king2.scs.fsu.edu/CEBProjects/awty/awty_start.php) [88]. Trees were visualized with Dendroscope v3.2.10 [89] and final tree editing was done using iTOL [90].

\section{Protein localization predictions}

Sub-cellular localization prediction was done using both manual and automated methods: PredAlgo (https://giavap-genomes.ibpc.fr/cgi-bin/predalgodb.perl?page=main) was used for the prediction of transit peptides in Archaeplastida algae [91], Hectar (http://webtools.sb-roscoff.fr/ tool_runner?tool_id=toolsheddev.sb-roscoff.fr\%2Frepos\%2 Flgueguen\%2Fhectar_3_0\%2Fabims_hectar\%2F1.0) was used for Heterokonts [92]. In addition, a manual method was used for organisms containing secondary plastids, as described elsewhere [93, 94]. Briefly, sequences were first analyzed using SignalP (http://www.cbs.dtu.dk/services/ SignalP/) [95]. For those sequences with positive prediction of endoplasmic reticulum (ER) trafficking, the signal peptides were manually removed and the resulting sequences were further analyzed using TargetP [95] to test for the presence of plastid targeting peptides.

\section{Structural predictions}

Important amino acids in C. reinhardtii DGAT3 were determined from the literature $[25,39]$ as well as via blastp, using the delta-blast algorithm in the NCBI website (https:// blast.ncbi.nlm.nih.gov/Blast.cgi). C. reinhardtii DGAT3 three-dimensional (3D) modeling and secondary structure prediction were done using Phyre2 [96] and I-Tasser [97] with default settings. Hydrophobicity analyses of C. reinhardtii DGAT1, DGAT2 and DGAT3 were done with ProtScale from the ExPASy web server [98] and the Kyte \& Doolittle amino acid hydropathicity scale [99] with a window size of nine for hydrophobic region identification. Transmembrane segment analysis was done using TMHMM v2.2 [100].

\section{Expression of C. reinhardtii DGAT3 in Escherichia coli}

The C. reinhardtii DGAT3 coding region was amplified by PCR using the following oligonucleotides: dgat 3 Forward 5' CATATGGCCTCTTTCGGCCTGCTGG 3' and dgat3 Reverse 5' GGATCCTTACGCCACTAGCTGATGC 3'. PCR products were cloned into Zero Blunt ${ }^{\circ}$ $\mathrm{TOPO}^{\circ}$ PCR Cloning Kit (Life Technologies, Carlsbad, CA, USA), further subcloned into pET19b (Novagen, Madison, WI, USA) and transformed into Escherichia coli BL21. Protein induction was done according to the Novagen pET system manual. Briefly, $500 \mu \mathrm{l}$ of overnight $E$. coli cultures (grown in LB with $100 \mu \mathrm{g} \mathrm{ml}^{-1}$ ampicillin at $28^{\circ}$ C) were added to fresh LB with the same antibiotic. Cultures were grown at $37^{\circ} \mathrm{C}$ to an $\mathrm{OD}$ of 0.6 and induced with $1 \mathrm{mM}$ IPTG for $4 \mathrm{~h}$. Cells were harvested by centrifugation at 10,000 $\times \mathrm{g}$ for $20 \mathrm{~min}$. For lipid analysis, $0.1 \%$ sodium oleate and $0.1 \%$ sodium gluconate were added to the induced cells $4 \mathrm{~h}$ after IPTG addition.

\section{Generation of anti-C. reinhardtii DGAT3 polyclonal antisera}

Histidine-tagged C. reinhardtii DGAT3 was purified using Ni-NTA agarose according to the manufacturer's instructions (QIA expressionist; Qiagen, Valencia, CA, USA). Briefly, cells were lysed by sonication in lysis buffer $(20 \mathrm{mM}$ Tris- $\mathrm{HCl} \mathrm{pH} \mathrm{8,} 0.5 \mathrm{M} \mathrm{NaCl}, 5 \mathrm{mM}$ imidazole, $1 \mathrm{mM}$ PMSF) and cell debris were eliminated by centrifugation at 10,000 $\times$ g for $20 \mathrm{~min}$. Supernatants were batch-incubated with the Ni-NTA resin for $1 \mathrm{~h}$ at $4{ }^{\circ} \mathrm{C}$ and the slurry was transferred 
to a plastic column. Three consecutive washes with lysis buffer were done with the following imidazole concentrations: 5, 20 and $60 \mathrm{mM}$. The $6 x$ His-DGAT3 protein was eluted in lysis buffer at $100 \mathrm{mM}$ imidazole, concentrated to $1 \mathrm{mg} / \mathrm{ml}$ and dialyzed into physiological saline solution $(0.85 \% \mathrm{NaCl})$ to generate rabbit polyclonal antibodies. To this aim, two rabbits were immunized subcutaneously with $500 \mathrm{ug}$ of protein mixed with equal volumen of complete Freund's adjuvant on days $0,7,16$ and with incomplete adjuvant on day 25 and 35 . Afterwards, the animals were anesthetized and sacrificed. The immune sera were frozen at $-20{ }^{\circ} \mathrm{C}$ and used for DGAT3 detection. All the procedures were performed according to the local institutional animal care (CICUAL, Universidad de Buenos Aires, Argentina).

\section{Protein electrophoresis and Western Blot analysis}

For protein preparations, E. coli cells were sonicated and centrifuged at $10,000 \times \mathrm{g}$ for $20 \mathrm{~min}$. Proteins in the supernatant were quantified using the bisinconinic acid reagent (Sigma, St. Louis, MO), separated by 12\% SDSPAGE and transferred to nitrocellulose (Genesee Scientific, San Diego, CA). Rabbit polyclonal antisera against C. reinhardtii DGAT3 were incubated overnight at $4{ }^{\circ} \mathrm{C}$. Alkaline phosphatase-labeled anti-rabbit secondary antibodies were used. Protein gel blots were developed using nitroblue tetrazolium and 5-bromo-4-chloro-3-indolyl phosphate (Sigma).

\section{Lipid extraction and thin layer chromatography analysis}

For lipid analysis, cell pellets were sonicated and extracted with methanol:chloroform (1:1). Thin layer chromatography (TLC) analysis was done in silica plates with a neutral lipid solvent system (hexane:diethyl ether:formic acid, 80:20: 2), loading the volume of lipids corresponding to $2.5 \mathrm{mg}$ of protein. A mix of wax esters and total lipid extract from rat liver were used as standards. Plates were developed using the sulfuric acid charring method. Briefly, plates were sprayed with a $8 \% \mathrm{CuSO}_{4}$ $(\mathrm{w} / \mathrm{v}), 10 \% \mathrm{H}_{3} \mathrm{PO}_{4}(\mathrm{v} / \mathrm{v})$ solution and the lipids visualized as black carbon depots formed after heating the plates at $180{ }^{\circ} \mathrm{C}$ for $1 \mathrm{~h}$.

\section{Additional files}

Additional file 1: Sources of the proteome files used for TAG pathway biocomputational HHMER iterative profiling. (XLSX $20 \mathrm{~kb}$ )

Additional file 2: Number of LCAT superfamily homologous sequences identified in algae by HMMER iterative data mining. (XLSX 8 kb)

Additional file 3: Full-length DGAT2 sequences identified in the selected algal species. (XLSX $10 \mathrm{~kb}$ )

Additional file 4: Transmembrane segment prediction of algal DGAT2 sequences. (PDF $1069 \mathrm{~kb})$

Additional file 5: Hydrophobicity and transmembrane segment analysis of C. reinhardtii DGATs. (PDF $418 \mathrm{~kb}$ )
Additional file 6: Phylogenetic analysis of the LCAT superfamily in algae and other representative taxa. (TXT $49 \mathrm{~kb}$ )

Additional file 7: Phylogenetic analysis of the MBOAT superfamily in algae and other representative taxa. (TXT $68 \mathrm{~kb}$ )

Additional file 8: Phylogenetic analysis of the DGAT2 family in algae and other representative taxa. (TXT $63 \mathrm{~kb}$ )

Additional file 9: Phylogenetic analysis of thiorredoxin-like ferredoxin domains in algae and other representative taxa. (TXT $101 \mathrm{~kb}$ )

Additional file 10: Phylogenetic analysis of DGAT3 and related proteins in algae and other representative taxa. (TXT $24 \mathrm{~kb}$ )

\section{Acknowledgements}

We thank Dr. Matías Mora for providing an evolutionary perspective to our DGAT3 results. We also thank Dr. Héctor Alvarez for critical input on our TLC analyses.

\section{Funding}

This work was supported by funds to MVB from the Argentinean Consejo Nacional de Investigaciones Científicas y Tecnológicas (CONICET-PIP 11420110100090) and from Agencia Nacional de Promoción Científica y Tecnológica (ANPCyT, PICT-2013-2122). MVB, CB, MBP, GRF, NS and SEM are CONICET Researchers.

\section{Availability of data and material}

The proteome files analyzed in this work are publicly available at the websites listed in Additional file 1. The raw data generated through our analyses are available in Additional files 1, 2, 3, 4, 5, 6, 7, 8, 9, 10.

\section{Authors' contributions}

CB took part in the computational analyses, TLC analyses, experimental design, data interpretation and manuscript writing and edition. MBP participated in the computational analyses, data interpretation and contributed to the writing and editing of the paper. GRF and NS did most of the TLC analyses. SEM had a major role in the preparation of anti-DGAT3 antisera. MVB participated in the computational analyses, in DGAT3 expression and purification, experimental design, data interpretation and had a major role in manuscript writing and editing. All authors read and approved the final manuscript.

Competing interests

The authors declare that they have no competing interests.

\section{Consent for publication}

Not applicable.

Ethics approval and consent to participate

Not applicable.

\section{Author details}

'Instituto de Energía y Desarrollo Sustentable, Comisión Nacional de Energía Atómica, Centro Atómico Bariloche, Av. Bustillo 9500, 8400S. C. de Bariloche, Río Negro, Argentina. ${ }^{2}$ Instituto de Investigaciones Bioquímicas de La Plata (INIBIOLP-CONICET-UNLP), Facultad de Ciencias Médicas, Universidad Nacional de La Plata, Calle 60 y 120 s/n, 1900 La Plata, Argentina.

${ }^{3}$ Universidad de Buenos Aires. CONICET Instituto de Investigaciones Cardiológicas (ININCA), - Laboratorio de Glico-Inmuno-Biología, Marcelo T. de Alvear 2270, C1122AAJ, Buenos Aires, Argentina. ${ }^{4}$ Instituto de Investigaciones Biológicas (IIB-CONICET-UNMdP), Facultad de Ciencias Exactas y Naturales, Universidad Nacional de Mar del Plata, CC 1245, 7600 Mar del Plata, Argentina.

Received: 24 November 2016 Accepted: 24 February 2017

Published online: 09 March 2017

\section{References}

1. Hu Q, Sommerfeld M, Jarvis E, Ghirardi M, Posewitz M, Seibert M, Darzins A. Microalgal triacylglycerols as feedstocks for biofuel production: perspectives and advances. Plant J. 2008;54:621-39.

2. Han S-F, Jin W-B, Tu R-J, Wu W-M. Biofuel production from microalgae as feedstock: current status and potential. Crit Rev Biotechnol. 2015;35:255-68. 
3. Passell H, Dhaliwal H, Reno M, Wu B, Ben Amotz A, Ivry E, Gay M, Czartoski T, Laurin L, Ayer N. Algae biodiesel life cycle assessment using current commercial data. J Environ Manage. 2013;129:103-11.

4. Du Z-Y, Benning C. Triacylglycerol accumulation in photosynthetic cells in plants and algae. Subcell Biochem. 2016;86:179-205.

5. Rodolfi L, Zittelli GC, Bassi N, Padovani G, Biondi N, Bonini G, Tredici MR. Microalgae for oil: strain selection, induction of lipid synthesis and outdoor mass cultivation in a low-cost photobioreactor. Biotechnol Bioeng. 2009;102:100-12.

6. Benvenuti G, Lamers PP, Breuer G, Bosma R, Cerar A, Wijfels RH, Barbosa MJ. Microalgal TAG production strategies: why batch beats repeated-batch. Biotechnol Biofuels. 2016;9:64

7. Chiu S-Y, Kao C-Y, Tsai M-T, Ong S-C, Chen C-H, Lin C-S. Lipid accumulation and $\mathrm{CO}_{2}$ utilization of Nannochloropsis oculata in response to $\mathrm{CO}_{2}$ aeration. Bioresour Technol. 2009;100:833-8.

8. Simionato D, Sforza E, Corteggiani Carpinelli E, Bertucco A, Giacometti GM, Morosinotto T. Acclimation of Nannochloropsis gaditana to different illumination regimes: effects on lipids accumulation. Bioresour Technol. 2011;102:6026-32.

9. Boyle NR, Page MD, Liu B, Blaby IK, Casero D, Kropat J, Cokus SJ, HongHermesdorf A, Shaw J, Karpowicz SJ, et al. Three acyltransferases and nitrogenresponsive regulator are implicated in nitrogen starvation-induced triacylglycerol accumulation in Chlamydomonas. J Biol Chem. 2012;287:15811-25.

10. Davidi L, Levin Y, Ben-Dor S, Pick U. Proteome analysis of cytoplasmatic and plastidic $\beta$-carotene lipid droplets in Dunaliella bardawil. Plant Physiol. 2015; 167:60-79.

11. Weng L-C, Pasaribu B, Ping Lin I, Tsai C-H, Chen C-S, Jiang P-L, Hastie LC, Waston TC, Isamu T, Belda CA, et al. Nitrogen deprivation induces lipid droplet accumulation and alters fatty acid metabolism in symbiotic dinoflagellates isolated from Aiptasia pulchella. Sci Rep. 2014;4:41-9.

12. Yang D, Song D, Kind T, Ma Y, Hoefkens J, Fiehn O, Li Y, Horsman M, Wu N, Lan C, et al. Lipidomic analysis of Chlamydomonas reinhardtii under nitrogen and sulfur deprivation. PLoS One. 2015;10, e0137948.

13. Adl SM, Simpson AGB, Lane CE, Lukeš J, Bass D, Bowser SS, Brown MW, Burki F, Dunthorn M, Hampl V, et al. The revised classification of eukaryotes. J Eukaryot Microbiol. 2012;59:429-93.

14. Janse I, van Rijssel M, van Hall P-J, Gerwig GJ, Gottshal J, Prins RA. The storage glucan of Phaeocystis globosa (Prymnesiophyceae) cells. J Phycol. 1996:32:382-7.

15. Van den Koornhuyse N, Libessart N, Delrue B, Zabawinski C, Decq A, Iglesias A, Carton A, Preiss J, Ball S. Control of starch composition and structure through substrate supply in the monocellular alga Chlamydomonas reinhardtii. J Biol Chem. 1996:271:16281-7.

16. Bigogno C, Khozin-Goldberg I, Boussiba S, Vonshak A, Cohen Z. Lipid and fatty acid composition of the green oleaginous alga Parietochloris incisa, the richest plant source of arachidonic acid. Phytochemistry. 2002;60:497-503.

17. Liu B, Benning C. Lipid metabolism in microalgae distinguishes itself. Curr Opin Biotechnol. 2013:24:300-9.

18. Merchant SS, Kropat J, Liu B, Shaw J, Warakanont J. TAG, You're it! Chlamydomonas as a reference organism for understanding algal triacylglycerol accumulation. Curr Opin Biotechnol. 2012;23:352-63.

19. Bell RM, Coleman RA. Enzymes of glycerolipid synthesis in eukaryotes. Annu Rev Biochem. 1980;49:459-87.

20. Yamashita S, Hosaka K, Taketo M, Numa S. Distribution of glycerolipid-synthesizing enzymes in the subfractions of rat liver microsomes. FEBS Lett. 1973;29:235-8.

21. Shockey JM, Gidda SK, Chapital DC, Kuan J-C, Dhanoa PK, Bland JM, Rothstein SJ, Mullen RT, Dyer JM. Tung tree DGAT1 and DGAT2 have nonredundant functions in triacylglycerol biosynthesis and are localized to different subdomains of the endoplasmic reticulum. Plant Cell. 2006;18:2294-313.

22. Cases S, Smith SJ, Zheng YW, Myers HM, Lear SR, Sande E, Novak S, Collins C, Welch CB, Lusis AJ, et al. Identification of a gene encoding an acyl CoA: diacylglycerol acyltransferase, a key enzyme in triacylglycerol synthesis. Proc Natl Acad Sci U S A. 1998;95:13018-23.

23. Cases S, Stone SJ, Zhou P, Yen E, Tow B, Lardizabal KD, Voelker T, Farese RV. Cloning of DGAT2, a second mammalian diacylglycerol acyltransferase, and related family members. J Biol Chem. 2001;276:38870-6.

24. Rani SH, Krishna THA, Saha S, Negi AS. Defective in Cuticular Ridges (DCR) of Arabidopsis thaliana, a gene associated with surface cutin formation, encodes a soluble diacylglycerol acyltransferase. J Biol Chem. 2010;285:38337-47.

25. Rani SH, Saha S, Rajasekharan R. A soluble diacylglycerol acyltransferase is involved in triacylglycerol biosynthesis in the oleaginous yeast Rhodotorula glutinis. Microbiology. 2013;159(Pt 1):155-66.
26. Durrett TP, McClosky DD, Tumaney AW, Elzinga DA, Ohlrogge J, Pollard M. A distinct DGAT with sn-3 acetyltransferase activity that synthesizes unusual, reduced-viscosity oils in Euonymus and transgenic seeds. Proc Natl Acad Sci U S A. 2010;107:9464-9.

27. Dahlqvist A, Stahl U, Lenman M, Banas A, Lee M, Sandager L, Ronne H, Stymne S. Phospholipid:diacylglycerol acyltransferase: an enzyme that catalyzes the acyl-CoA-independent formation of triacylglycerol in yeast and plants. Proc Natl Acad Sci U S A. 2000;97:6487-92.

28. Ståhl U, Carlsson AS, Lenman M, Dahlqvist A, Huang B, Bana W, Bana A, Stymne S. Cloning and functional characterization of a phospholipid: diacylglycerol acyltransferase from Arabidopsis. Plant Physiol. 2004;135:1324-35.

29. Cao H. Structure-function analysis of diacylglycerol acyltransferase sequences from 70 organisms. BMC Res Notes. 2011;4:249.

30. Liu Q, Siloto RMP, Lehner R, Stone SJ, Weselake RJ. Acyl-CoA:diacylglycerol acyltransferase: molecular biology, biochemistry and biotechnology. Prog Lipid Res. 2012;51:350-77.

31. Turchetto-Zolet AC, Maraschin FS, de Morais GL, Cagliari A, Andrade CM, Margis-Pinheiro M, Margis R. Evolutionary view of acyl-CoA diacylglycerol acyltransferase (DGAT), a key enzyme in neutral lipid biosynthesis. BMC Evol Biol. 2011;11:263-77.

32. Wang $P$, Wang Z, Dou Y, Zhang X, Wang M, Tian X. Genome-wide identification and analysis of membrane-bound O-acyltransferase (MBOAT) gene family in plants. Planta. 2013;238:907-22.

33. Pan X, Peng FY, Weselake RJ. Genome-wide analysis of Phospholipid: Diacylglycerol Acyltransferase (PDAT) genes in plants reveals the eudicotwide PDAT gene expansion and altered selective pressures acting on the core eudicot PDAT paralogs. Plant Physiol. 2015;167:887-904.

34. Chen JE, Smith AG. A look at diacylglycerol acyltransferases (DGATs) in algae. J Biotechnol. 2012;162:28-39.

35. Chen C-X, Sun Z, Cao H-S, Fang F-L, Ouyang L-L, Zhou Z-G. Identification and characterization of three genes encoding acyl-CoA: diacylglycerol acyltransferase (DGAT) from the microalga Myrmecia incisa Reisigl. Algal Res. 2015;12:280-8.

36. Skewes-Cox P, Sharpton TJ, Pollard KS, DeRisi JL. Profile hidden Markov models for the detection of viruses within metagenomic sequence data. PLoS One. 2014;9, e105067.

37. Madera M, Gough J. A comparison of profile hidden Markov model procedures for remote homology detection. Nucleic Acids Res. 2002; 30:4321-8.

38. Alam I, Dress A, Rehmsmeier M, Fuellen G, Fitch WM. Comparative homology agreement search: an effective combination of homology-search methods. Proc Natl Acad Sci U S A. 2004;101:13814-9.

39. Saha S, Enugutti B, Rajakumari S, Rajasekharan R. Cytosolic triacylglycerol biosynthetic pathway in oilseeds. molecular cloning and expression of peanut cytosolic diacylglycerol acyltransferase. Plant Physiol. 2006; 141(August):1533-43.

40. Cao H, Shockey JM, Klasson KT, Chapital DC, Mason CB, Scheffler BE. Developmental regulation of diacylglycerol acyltransferase family gene expression in tung tree tissues. PLoS One. 2013;8, e76946.

41. Banaś A, Carlsson AS, Huang B, Lenman M, Bana W, Lee M, Noiriel A, Benveniste P, Schaller H, Bouvier-Navé P, Stymne S. Cellular sterol ester synthesis in plants is performed by an enzyme (phospholipid:sterol acyltransferase) different from the yeast and mammalian Acyl-CoA:sterol acyltransferases. J Biol Chem. 2005;280.

42. Chen G, Greer MS, Lager I, Lindberg Yilmaz J, Mietkiewska E, Carlsson AS, Stymne S. Weselake RJ. Identification and characterization of an LCAT-like Arabidopsis thaliana gene encoding a novel phospholipase A. FEBS Lett. 2012;586:373-7.

43. Peelman F, Vinaimont N, Verhee A, Vanloo B, Verschelde JL, Labeur C, SeguretMace S, Duverger N, Hutchinson G, Vandekerckhove J, et al. A proposed architecture for lecithin cholesterol acyl transferase (LCAT): identification of the catalytic triad and molecular modeling. Protein Sci. 1998;7:587-99.

44. Peelman F, Verschelde JL, Vanloo B, Ampe C, Labeur C, Tavernier J, Vandekerckhove J, Rosseneu M. Effects of natural mutations in lecithin:cholesterol acyltransferase on the enzyme structure and activity. J Lipid Res. 1999:40:59-69.

45. Chang TY, Chang CC, Lin S, Yu C, Li BL, Miyazaki A. Roles of acyl-coenzyme A:cholesterol acyltransferase-1 and -2. Curr Opin Lipidol. 2001;12:289-96.

46. Yang H, Bard M, Bruner DA, Gleeson A, Deckelbaum RJ, Aljinovic G, Pohl TM, Rothstein R, Sturley SL. Sterol esterification in yeast: a two-gene process. Science. 1996;272:1353-6.

47. Hung $\mathrm{C}-\mathrm{H}, \mathrm{H}$ o M-Y, Kanehara K, Nakamura Y. Functional study of diacylglycerol acyltransferase type 2 family in Chlamydomonas reinhardtii. FEBS Lett. 2013;587:2364-70. 
48. Deng XD, Gu B, Li YJ, Hu XW, Guo JC, Fei XW. The roles of acyl-CoA: diacylglycerol acyltransferase 2 genes in the biosynthesis of triacylglycerols by the green alga Chlamydomonas reinhardtii. Mol Plant. 2012;5:945-7.

49. Iwai M, Ikeda K, Shimojima M, Ohta H. Enhancement of extraplastidic oil synthesis in Chlamydomonas reinhardtii using a type-2 diacylglycerol acyltransferase with a phosphorus starvation-inducible promoter. Plant Biotechnol J. 2014;12:808-19.

50. Raux E, Leech HK, Beck R, Schubert HL, Santander PJ, Roessner CA, Scott Al, Martens $\mathrm{JH}$, Jahn D, Thermes $\mathrm{C}$, et al. Identification and functional analysis of enzymes required for precorrin-2 dehydrogenation and metal ion insertion in the biosynthesis of sirohaem and cobalamin in Bacillus megaterium. Biochem J. 2003;370(Pt 2):505-16.

51. Machray GC, Burch L, Hedley PE, Davies H, Waugh R. Characterisation of a complementary DNA encoding a novel plant enzyme with sucrolytic activity. FEBS Lett. 1994;354:123-7.

52. Appel J, Schulz R. Sequence analysis of an operon of a NAD(P)-reducing nickel hydrogenase from the cyanobacterium Synechocystis sp. PCC 6803 gives additional evidence for direct coupling of the enzyme to $\mathrm{NAD}(\mathrm{P}) \mathrm{H}$ dehydrogenase (complex I). Biochim Biophys Acta. 1996;1298:141-7.

53. Takeuchi K, Reue K. Biochemistry, physiology, and genetics of GPAT, AGPAT, and lipin enzymes in triglyceride synthesis. Am J Physiol Endocrinol Metab. 2009;296:E1195-209.

54. Fan J, Andre C, Xu C. A chloroplast pathway for the de novo biosynthesis of triacylglycerol in Chlamydomonas reinhardtii. FEBS Lett. 2011;585:1985-91.

55. Goodson C, Roth R, Wang ZT, Goodenough U. Structural correlates of cytoplasmic and chloroplast lipid body synthesis in Chlamydomonas reinhardtii and stimulation of lipid body production with acetate boost. Eukaryot Cell. 2011;10:1592-606.

56. Goold HD, Cuiné $S$, Legeret $B$, Liang $Y$, Brugière $S$, Auroy $P$, Javot $H$, Tardif $M$, Jones BJ, Beisson F, et al. Saturating light induces sustained accumulation of oil in plastidal lipid droplets in Chlamydomonas reinhardtii. Plant Physiol. 2016;171:2406-17.

57. Kalscheuer R, Steinbüchel A. A novel bifunctional wax ester synthase/acyl-CoA: Diacylglycerol acyltransferase mediates wax ester and triacylglycerol biosynthesis in Acinetobacter calcoaceticus ADP1. J Biol Chem. 2003;278:8075-82.

58. Kalscheuer R, Stoveken T, Luftmann H, Malkus U, Reichelt R, Steinbuchel A. Neutral lipid biosynthesis in engineered Escherichia coli: Jojoba oil-like wax esters and fatty acid butyl esters. Appl Environ Microbiol. 2006;72:1373-9.

59. Waltermann M, Luftmann $H$, Baumeister D, Kalscheuer $R$, Steinbuchel A. Rhodococcus opacus strain PD630 as a new source of high-value single-cell oil? Isolation and characterization of triacylglycerols and other storage lipids. Microbiology. 2000;146:1143-9.

60. Newman DK, Kolter R. A role for excreted quinones in extracellular electron transfer. Nature. 2000;405:94-7.

61. Alvarez HM, Steinbüchel A. Triacylglycerols in prokaryotic microorganisms. Appl Microbiol Biotechnol. 2002;60:367-76.

62. Waltermann M, Steinbuchel A. Neutral lipid bodies in prokaryotes: recent insights into structure, formation, and relationship to eukaryotic lipid depots. J Bacteriol. 2005;187:3607-19.

63. Karpowicz SJ, Prochnik SE, Grossman AR, Merchant SS. The GreenCut2 resource, a phylogenomically derived inventory of proteins specific to the plant lineage. J Biol Chem. 2011;286:21427-39.

64. Yeh AP, Chatelet C, Soltis SM, Kuhn P, Meyer J, Rees DC. Structure of a thioredoxinlike [2Fe-2S] Ferredoxin from Aquifex aeolicus. J Mol Biol. 2000;300:587-95.

65. Chatelet C, Gaillard J, Pétillot Y, Louwagie M, Meyer J. A [2Fe-2S] protein from the hyperthermophilic bacterium Aquifex Aeolicus. Biochem Biophys Res Commun. 1999;261:885-9.

66. Zu Y, Di Bernardo S, Yagi T, Hirst J. Redox properties of the [2Fe-2S] center in the $24 \mathrm{kDa}$ (NQO2) Subunit of NADH:ubiquinone oxidoreductase (Complex I). Biochemistry. 2002;41:10056-69.

67. Burgdorf T, van der Linden E, Bernhard M, Yin QY, Back JW, Hartog AF, Muijsers AO, de Koster CG, Albracht SPJ, Friedrich B. The soluble NAD + -Reducing [NiFe]-hydrogenase from Ralstonia eutropha H16 consists of six subunits and can be specifically activated by NADPH. J Bacteriol. 2005;187:3122-32.

68. Goodenough U, Blaby I, Casero D, Gallaher SD, Goodson C, Johnson S, Lee J-H, Merchant SS, Pellegrini M, Roth $\mathrm{R}$, et al. The path to triacylglyceride obesity in the sta6 strain of Chlamydomonas reinhardtii. Eukaryot Cell. 2014;13:591-613.

69. Terashima M, Specht M, Hippler M. The chloroplast proteome: a survey from the Chlamydomonas reinhardtii perspective with a focus on distinctive features. Curr Genet. 2011;57:151-68.
70. Yoon K, Han D, Li Y, Sommerfeld M, Hu Q. Phospholipid:diacylglycerol acyltransferase is a multifunctional enzyme involved in membrane lipid turnover and degradation while synthesizing triacylglycerol in the unicellular green microalga Chlamydomonas reinhardtii. Plant Cell. 2012;24: 3708-24.

71. Davidi L, Shimoni E, Khozin-Goldberg I, Zamir A, Pick U. Origin of betacarotene-rich plastoglobuli in Dunaliella bardawil. Plant Physiol. 2014;164: 2139-56.

72. Sakaki T, Kondo N, Yamada M. Pathway for the synthesis of triacylglycerols from monogalactosyldiacylglycerols in ozone-fumigated spinach leaves. Plant Physiol. 1990;94:773-80.

73. Kaup MT, Froese CD, Thompson JE. A role for diacylglycerol acyltransferase during leaf senescence. Plant Physiol. 2002;129:1616-26.

74. Martin BA, Wilson RF. Subcellular localization of triacylglycerol synthesis in spinach leaves. Lipids. 1984;19:117-21.

75. Li X, Moellering ER, Liu B, Johnny C, Fedewa M, Sears BB, Kuo M-H, Benning C. A galactoglycerolipid lipase is required for triacylglycerol accumulation and survival following nitrogen deprivation in Chlamydomonas reinhardtii. Plant Cell. 2012;24:4670-86.

76. The UniProt Consortium. UniProt: a hub for protein information. Nucleic Acids Res. 2014;43(Database issue):D204-12.

77. Eddy SR. Profile hidden Markov models. Bioinformatics. 1998;14:755-63.

78. Katoh K, Misawa K, Kuma K, Miyata T. MAFFT: a novel method for rapid multiple sequence alignment based on fast Fourier transform. Nucleic Acids Res. 2002;30:3059-66.

79. Larsson A. Aliview: A fast and lightweight alignment viewer and editor for large datasets. Bioinformatics. 2014;30:3276-8.

80. Nicholas K, Nicholas H, Deerfield D. GeneDoc: analysis and visualization of genetic variation. EMBnet News. 1997:4:14.

81. Fu L, Niu B, Zhu Z, Wu S, Li W. CD-HIT: accelerated for clustering the nextgeneration sequencing data. Bioinformatics. 2012;28:3150-2.

82. Criscuolo A, Gribaldo S. BMGE (Block Mapping and Gathering with Entropy): a new software for selection of phylogenetic informative regions from multiple sequence alignments. BMC Evol Biol. 2010;10:210.

83. Crooks GE, Hon G, Chandonia J-M, Brenner SE. WebLogo: a sequence logo generator. Genome Res. 2004;14:1188-90.

84. Guindon S, Dufayard JF, Lefort V, Anisimova M, Hordijk W, Gascuel O. New algorithms and methods to estimate maximum-likelihood phylogenies: Assessing the performance of PhyML 3.0. Syst Biol. 2010;59:307-21.

85. Minh BQ, Nguyen MAT, von Haeseler A. Ultrafast approximation for phylogenetic bootstrap. Mol Biol Evol. 2013;30:1188-95.

86. Trifinopoulos J, Nguyen L-T, von Haeseler A, Minh BQ. W-IQ-TREE: a fast online phylogenetic tool for maximum likelihood analysis. Nucleic Acids Res. 2016;44:W232-5.

87. Ronquist F, Teslenko M, Van Der Mark P, Ayres DL, Darling A, Höhna S, Larget B, Liu L, Suchard MA, Huelsenbeck JP. Mrbayes 3.2: efficient bayesian phylogenetic inference and model choice across a large model space. Syst Biol. 2012;61:539-42.

88. Nylander JAA, Wilgenbusch JC, Warren DL, Swofford DL. AWTY (are we there yet?): a system for graphical exploration of MCMC convergence in Bayesian phylogenetics. Bioinformatics. 2008;24:581-3.

89. Huson DH, Scornavacca C. Dendroscope 3: an interactive tool for rooted phylogenetic trees and networks. Syst Biol. 2012;61:1061-7.

90. Letunic I, Bork P. Interactive tree of life (iTOL) v3: an online tool for the display and annotation of phylogenetic and other trees. Nucleic Acids Res. 2016;44:W242-5.

91. Tardif M, Atteia A, Specht M, Cogne G, Rolland N, Brugière S, Hippler M, Ferro M, Bruley C, Peltier G, et al. PredAlgo: a new subcellular localization prediction tool dedicated to green algae. Mol Biol Evol. 2012;29:3625-39.

92. Gschloessl B, Guermeur Y, Cock JM. HECTAR: a method to predict subcellular targeting in heterokonts. BMC Bioinformatics. 2008;9:393.

93. Jiroutová K, Horák A, Bowler C, Oborník M. Tryptophan biosynthesis in stramenopiles: eukaryotic winners in the diatom complex chloroplast. J Mol Evol. 2007:65:496-511.

94. Beligni MV, Bagnato C, Prados MB, Bondino H, Laxalt AM, Munnik T, Ten Have A. The diversity of algal phospholipase $D$ homologs revealed by biocomputational analysis. J Phycol. 2015;51:943-62.

95. Emanuelsson $\mathrm{O}$, Brunak $\mathrm{S}$, von Heijne $\mathrm{G}$, Nielsen $\mathrm{H}$. Locating proteins in the cell using TargetP, SignalP and related tools. Nat Protoc. 2007;2:953-71.

96. Kelley. The Phyre2 web portal for protein modelling, prediction, and analysis. Nat Protoc. 2015;10:845-58. 
97. Yang J, Yan R, Roy A, Xu D, Poisson J, Zhang Y. The I-TASSER Suite: protein structure and function prediction. Nat Methods. 2015;12:7-8.

98. Gasteiger E, Hoogland C, Gattiker A, Duvaud S, Wilkins MR, Appel RD, Bairoch A. Protein identification and analysis tools on the ExPASy server. In: Walker JM, editor. The proteomics protocols handbook. Totowa: Humana Press Inc; 2005. p. 571-607.

99. Kyte J, Doolittle RF. A simple method for displaying the hydropathic character of a protein. J Mol Biol. 1982;157:105-32.

100. Krogh A, Larsson B, von Heijne G, Sonnhammer EL. Predicting transmembrane protein topology with a hidden Markov model: application to complete genomes. J Mol Biol. 2001;305:567-80.

Submit your next manuscript to BioMed Central and we will help you at every step:

- We accept pre-submission inquiries

- Our selector tool helps you to find the most relevant journal

- We provide round the clock customer support

- Convenient online submission

- Thorough peer review

- Inclusion in PubMed and all major indexing services

- Maximum visibility for your research

Submit your manuscript at www.biomedcentral.com/submit
Biomed Central 